\title{
3-D Hybrid VLC-RF Indoor IoT Systems with Light Energy Harvesting
}

\author{
Gaofeng Pan, Member, IEEE, Hongjiang Lei, Member, IEEE, Zhiguo Ding, Senior Member, IEEE, and Qiang Ni, \\ Senior Member, IEEE
}

\begin{abstract}
In this paper, a 3-dimensional (3-D) hybrid visible light communication (VLC)-radio frequency (RF) indoor internet of things system with spatially random terminals with one photodiode (e.g., indoor sensors: temperature sensors, humidity sensors, and indoor air quality sensors) is considered. Specifically, homogeneous Poisson point process is adopted to model to the distribution of the terminals, which means that the number of the terminals obeys Poisson distribution, and the positions of the terminals are uniformly distributed. VLC and RF communications are employed over downlink and uplink, respectively. Meanwhile, the terminals are designed to harvest the energy from the light emitted by the light-emitting diode over the downlink, which is used for the transmissions over the uplink. The light energy harvesting model is considered after introducing the line of sight propagation model for VLC. Then, the outage performance has been studied for the VLC downlink and non-orthogonal multiple access schemes over the RF uplink, respectively, by using stochastic geometry theory, while considering the randomness of the number of the terminals, and all terminals are spatially and randomly distributed in the 3-D room and all RF uplinks follow Rician fading. Finally, the approximated analytical expressions for the outage probability are derived and verified through Monte Carlo simulations.
\end{abstract}

Index Terms-Light energy harvesting, non-orthogonal multiple access, outage probability, radio frequency, Rician fading, stochastic geometry, visible light communication.

\section{INTRODUCTION}

Recently, visible light communication (VLC) has been proposed and recognized as an alternative for traditional wireless radio-frequency (RF) communications in indoor scenarios, which can offer a potential on high-speed transmission to satisfy the growing demand for high data rates $[1,2]$. Specifically, in light emitting diode (LED) based VLC systems, LED is adopted as optical sources to convert the electrical signal to the modulated optical signals, while photodiodes (PDs) are

Manuscript received September 27, 2018; revised February 10 2019; accepted March 27 2019. This research was supported in part by Fundamental Science and Frontier Technology Research Plan of Chongqing under Grant cstc2017jcyjAX0204. The work of Q. Ni was supported by the Royal Society project IEC170324. The work of Z. Ding was supported by the UK EPSRC under grant number EP/P009719/2 and by H2020-MSCA-RISE-2015 under grant number 690750 .The associate editor coordinating the review of this paper and approving it for publication was ***. (Corresponding author: Gaofeng Pan.)

G. Pan is with School of Information and Electronics, Beijing Institute of Technology, Beijing 100081, P. R. China. e-mail: Gaofeng.Pan.CN@ieee.org.

H. Lei is with School of Communication and Information Engineering, Chongqing University of Posts and Telecommunications, Chongqing 400065, China.

Z. Ding is with School of Electrical and Electronic Engineering, University of Manchester, Manchester, M13 9PL, U.K.

Q. Ni is with School of Computing and Communications, Lancaster University, Lancaster, LA1 4WA, U.K. used as the detector at the receivers to convert the optical power back into electrical current for signal processing.

On the other hand, energy harvesting (EH) from the surrounding environments has been regarded as a promising and practical way to prolong the lifetime of power-constrained systems, e.g., wireless sensor networks (WSNs), wireless personal networks and blacktooth networks, which always work under extremely low duty cycles $[3,4]$. The energy sources that can be harvested are diverse, especially for WSNs [5] and, among those, the level of indoor lighting has been proved to be enough to power electronic applications $[6,7]$. Light EH can be offered by VLC, while supporting high data-rate links without producing any electromagnetic pollution, as well as meeting the requirements of the eye safety regulations. Moreover, benefiting from the ever-increasing popularity of solid-state lighting, light EH can be realized economically, as existing illumination facilities can be utilized.

Generally, there are two main schemes adopted to harvest energy from the light: solar panel and photodiodes (PDs) [8, 9]. Though solar panels capture the optical rays to electrical signals without any external power supply [10-13], it may inevitably lead to much sizeable hardware and increased cost. In [10], a solar panel based light EH receiver was proposed, where the alternating current (AC) and direct current (DC) components of the received light signal are split for information decoding and $\mathrm{EH}$, respectively. The authors of [11] conducted experiments on solar cell efficiency under several types of indoor environment using a typical commercial solar panel, and found that, within sunshine illuminated rooms or under direct light exposure from a lamp, the power requirement for cell phone charging can be satisfied or provided to some extent. Actually, the constructions of PDs and photovoltaic cells are essentially same as both of them have P-N junctions. Therefore, integrated EH PDs were designed to harvest energy for subdermal biomedical devices [14], substrate/well photodiodes fabricated in standard complimentary metal-oxidesemiconductor were presented to power self-powered image sensors [15], and GaAs based indoor EH was proposed to power millimeter-scale systems [16]. Inspired by these hardware models, one can see that VLC is able to offer a potential of harvesting energy from the lights, e.g., solar panel based light EH [10,11] and PD based light EH [15, 16], for indoor energyconstrained systems, e.g., indoor IoT systems. Therefore, light EH has been considered during designing the VLC systems. Simultaneous lightwave information and power transfer was proposed for IoT systems to make use of VLC signal in [17]. In [18], light EH was designed at the relay to harvest energy 
for the data delivery between the relay and the destination. A light EH model was proposed for VLC systems in one of our previous works [19]. However, only the secrecy outage performance of the RF uplink was investigated in [19], while the device is distributed in a 2-dimensional (2-D) circle and no multiple access schemes have been considered.

Therefore, one can see that, though the power of VLC light signal is low, the proposed light EH can work well for indoor IoT systems. Because in IoT systems, the traffic load at each indoor IoT device, e.g., temperature sensors, humidity sensors, and indoor air quality sensors, is very low, which is normally on the order of tens of bits, even on the order of bits, resulting in quite tiny transmission time at IoT devices. Also, the work cycle of these indoor IoT devices will not as high as normal wireless terminals, as IoT devices always report their data one time every long duration. As inspired by the fact that light $\mathrm{EH}$ can provide the potentials to charge the sensors in indoor scenarios, VLC with EH enables operating monitoring and control with deeply embedded sensors, e.g., temperature, humidity, indoor air quality sensors, for next generation intelligent building management [20]- [21].

Hybrid VLC-RF systems, in which RF delivery is considered for the uplink, have been recently chosen to implement efficient VLC systems, as a power-hungry light source is normally impractical for the sensors with limited size and constrained battery capacity, and VLC over the uplink may produce glare, which is uncomfortable to human. Then, the traditional RF transmissions, e.g., wireless fidelity (Wi-Fi), are a suitable alternative for the uplink of indoor VLC systems. So far, VLC-RF systems have gained more and more attention in academia and industry [22], driven by the fact that the hybrid system allows the traditional RF to enhance the benefits of VLC with acceptable cost and no interference $[24,25]$.

It is clear that none of the above-mentioned works has considered or studied light EH for hybrid VLC-RF system. Moreover, recalling the works on light EH in VLC system [10]- [16], one can also easily find that no analytical model has been established, other than the proposed hardware designs.

Motivated by the above observations, this work aims at proposing and analyzing a 3D hybrid RF-VLC system with light EH for indoor IoT, where VLC and RF are deployed for the downlink and the uplink, respectively. In this system, all devices are uniformly deployed in a 3-dimensional (3-D) room, while the LED lights are placed in the center of the ceiling. At each device, light EH is conducted by using PDs and the harvested energy is adopted for the transmissions over the RF uplink. Specially, we focus on the outage performance of the targeted VLC-RF system. In practical, terminals, e.g., wireless sensors, are always distributed in the 3-D space of the room, not only be placed on the 2-D floor. Therefore, differing from [19] and [27] (where only 2-D circle was considered) and in order to reflect the actual distribution of the devices in indoor scenarios, stochastic geometry theory has been employed in this paper to deal with the randomness of the positions of the devices in a 3-D space.

To the best of our knowledge, this is the first work proposing advanced stochastic modeling analysis of 3-D hybrid VLCRF system with light EH. Main contributions of this work are listed as follows:

(1) Light energy harvesting is considered for indoor hybrid VLC-RF energy-constrained systems in this work, while existing studies mostly focus on RF signal energy harvesting.

(2) Differing from [19] and [27], in this work, we study the outage performance of the VLC downlink, while considering that devices are randomly deployed in a 3-D space.

(3) Though [19] and [26] have considered light EH, the secrecy outage of orthogonal multiple access RF uplink was considered. In this work, we investigated the outage of nonorthogonal multiple access (NOMA) scheme over the uplink, which has not been studied while considering the harvested energy over the uplink.

(4) The analysis method for the outage performance over Rician fading in 3-D space has been developed, while considering the randomness of the positions of the terminals and the light EH over the VLC downlink.

This paper is structured as follows. In Section II, the considered system model is presented. In Section III, the channel model for VLC and the proposed light EH model are presented; The outage analysis for the VLC downlink and RF uplink is presented in Section IV and V, respectively. In Section VI, the proposed analytical models are verified by Monte-Carlo simulations. Finally, the paper is concluded in Section VII.

\section{SYSTEM MODEL}

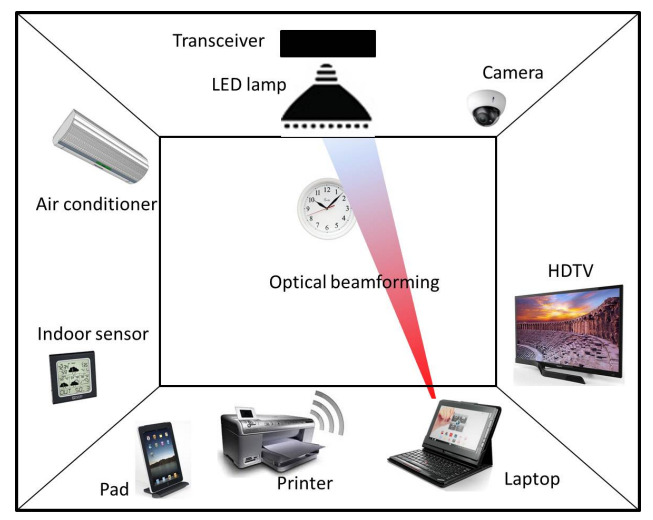

Fig. 1: 3-D indoor VLC-RF scenario

In this work, we consider a typical indoor IoT application scenario of the hybrid VLC-RF systems, as shown in Fig. 1. A group of $L(L \geq 1)$ LED lamps are placed in the center of the ceiling to insure room illumination according to the illumination standards to meet the human safety regulation, as well as to deliver data to the terminals (e.g., HDTV, printer, air conditioner, laptop and tablet), and indoor IoT devices (e.g., temperature sensors, humidity sensors, and indoor air quality sensors) placed in the room.

Moreover, the ever-increasing popularity of LED lights can also offer another important energy harvesting source: VLC signal. Therefore, in this paper we also assume that all IoT devices are with PD not only for the purposes of data communication but also with the aim of harvesting energy from the light emitted by the LED lamps. Then, the harvested energy 
can be used to transmit data to the RF receiver placed with the lamps over the RF uplink, as so to collect the data from each device. Specially, in our VLC-RF system shown in Fig. 1, identical LED lamps are adopted to provide data delivery to the devices, e.g., household appliances and indoor sensors, over the downlink. On the other hand, RF communication is adopted for the transmission from the devices to the RF transceiver to overcome the drawbacks arisen from adopting VLC over the uplink ${ }^{1}$.

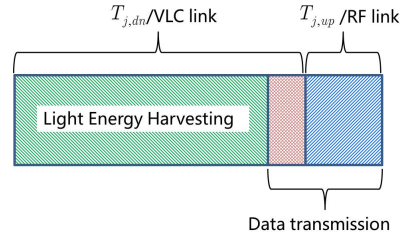

Fig. 2: Diagram of the information delivery in the target system

The information delivery between the device and the LED can be divided into three stages, as suggested by Fig. $2^{2}$ :

- Devices harvest energy from the lights emitted by the LED lamps, when there are no data transmissions between the LED lamps and the devices;

- During the data collection process, the LED lamps first transmit control signaling information to ask the devices to send back their data to the RF receiver over the RF uplink;

- Devices transmit their information back to the RF receiver at the LED lamps by using RF transmission technologies under NOMA scheme.

For tractability purpose, in this work we also treat the space of the room as a hemisphere, $\mathcal{V}$, with radius, $D$, where the LED lamps are located at the center of the sphere ${ }^{3}$. The $N(N \geq 1)$ devices can be modeled as a set of independent and identical uniformly distributed points in the hemisphere $\mathcal{V}$, denoted by $W_{j}$. Therefore, the distance between the device and the LED can be calculated from $W_{j}$, the pdf of which can be given by using Eq. (20) in [28] as

$$
f_{W_{j}}\left(w_{j}\right)=\frac{3}{2 \pi D^{3}}=\beta .
$$

In this paper, it is assumed that all devices and the the $\mathrm{RF}$ receiver are with a single antenna, and NOMA scheme is considered for the transmissions over the RF uplink to support concurrent transmissions over the uplink.

\section{LIGHT EH OVER VLC LINK}

In this section, we first introduce the channel modeling for VLC, and then the adopted light EH model is presented.

\footnotetext{
${ }^{1}$ The $\mathrm{RF}$ receiver is placed with LED lamps to realize the RF communication over the uplink.

${ }^{2}$ One can easily see that the information delivery over the VLC downlink is the prerequisite of the one over the RF uplink. Then, in the following sections we will respectively present the outage analysis for the VLC downlink and RF uplink to clearly show the system performance.

${ }^{3}$ The main difference between a hemisphere and a cuboid/cube room is that some spaces close to the floor of the room are omitted. As in practical scenarios indoor IoT devices are rarely placed in these spaces, the adopted modeling method is reasonable.
}

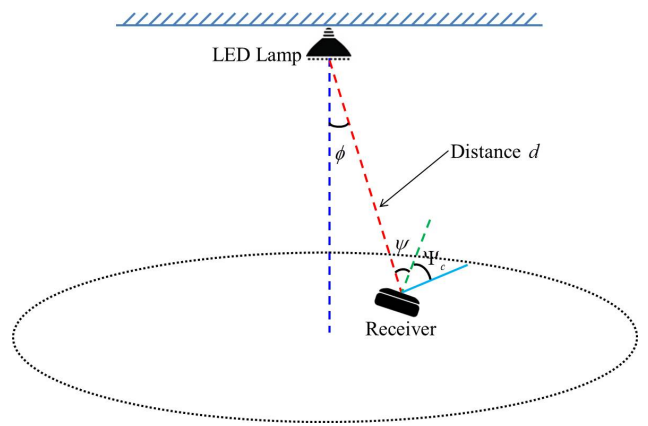

Fig. 3: 3-D LOS channel model

\section{A. LOS Propagation Model for VLC}

There is a line of sight (LOS) path from the LED to each node, and also a diffuse path via the reflections from surfaces within the room, as shown in Fig. 3.

The channel gain from the transmitter to the receiver is given by [29]- [31]

$$
h=\left\{\begin{array}{ll}
\frac{(m+1) A}{2 \pi d^{2}} \cos ^{m}(\phi) T_{s}(\psi) g(\psi) \cos \psi, & 0 \leq \psi \leq \Psi_{c} \\
0, & \psi>\Psi_{c}
\end{array},\right.
$$

where $d$ is the distance between the LED lamp and the receiver, $A$ is the area of the photodetector, $\phi$ is the transmitter viewing angle (also referred as irradiation angle), $\psi$ is the angle of incidence with respect to the receiver axis, $\Psi_{c}$ is the concentrator field of view (FOV), $T_{s}(\psi)$ denotes the gain of the optical filter adopted at the receiver, $m$ denotes the order of Lambertian emission, which is related to $\phi_{1 / 2}$ (the semiangle of the LED), the transmitter semi-angle (at half power), by $m=-\ln 2 / \ln \left(\cos \left(\phi_{1 / 2}\right)\right), g(\psi)$ denotes the gain of the non-imaging concentrator, which is given by Eq. (8) in [29] as follows

$$
g(\psi)=\left\{\begin{array}{ll}
\frac{p_{\text {in }}^{2}}{\sin ^{2}\left(\Psi_{c}\right)}, & 0 \leq \psi \leq \Psi_{c} \\
0, & \psi>\Psi_{c}
\end{array},\right.
$$

where $p_{\text {in }}$ is the internal refractive index of the optical concentrator adopted at the receiver front-end. In this work, it is assumed that the average optical transmit power $\left(P_{t}\right)$ and the area of the photodetector at all LED lamps are same, and the distances between the $j$ th $(1 \leq j \leq N)$ receiver and all LED lamps are same to simplify the following analysis.

Then, the received optical power from the $i$ th $(1 \leq i \leq L)$ LED lamp at the $j$ th device can be given as

$$
\begin{aligned}
P_{i j R} & =P_{t} h_{i j} \\
& =P_{t} \frac{\left(m_{i}+1\right) A}{2 \pi d_{i j}^{2}} \cos ^{m_{i}}\left(\phi_{i j}\right) T_{s}\left(\psi_{i j}\right) g\left(\psi_{i j}\right) \cos \left(\psi_{i j}\right),
\end{aligned}
$$

where $h_{i j}, m_{i}, \phi_{i j}, \psi_{i j}$ and $d_{j}$ are the parameters defined in (2) for the $i$ th LED lamp and the $j$ th device. It is obvious that $P_{i j R}$ is not only related to the distance between the $i$ th lamp and the $j$ th device, but also related to both the radiation pattern of the $i$ th lamp and the incidence angle of the $j$ th device. 
As suggested in [32]- [33], the RF power from the $i$ th lamp at the $j$ th device can be written as

$$
\begin{aligned}
P_{i j, R F} & =C_{j, R F} P_{i j R}{ }^{2} \\
& =\frac{C_{j, R F} P_{t}^{2}}{d_{i j}^{4}} G_{t}^{2}\left(\phi_{i j}\right) G_{r}^{2}\left(\psi_{i j}\right),
\end{aligned}
$$

where $C_{j, R F}$ is the power constant at the $j$ th device, which is related to the optical-to-electrical conversion efficiency and optical power constant given the following relation $\left(P_{j, R F}\right)^{\frac{1}{2}} \propto$ $i_{P D j} \propto P_{r j}, i_{P D j}$ is the current of the PD at the $j$ th device, $G_{t}\left(\phi_{i j}\right)$ and $G_{r}\left(\psi_{i j}\right)$ are the transmitting and receiving gains the radiation angle $\phi_{i j}$ and incidence angle $\psi_{i j}$, respectively, which can be given as [32]- [33]

$$
G_{t}\left(\phi_{i j}\right)=\cos ^{m_{i, t}}\left(\phi_{i j}\right)
$$

and

$$
G_{r}\left(\psi_{i j}\right)=\cos ^{m_{i j, r}}\left(\psi_{i j}\right),
$$

respectively, where $m_{i, t}$ and $m_{i j, r}$ are related to $\phi_{i, 1 / 2}$ and $\psi_{i j, 1 / 2}$, respectively, which are the $i$ th lamp and the $j$ th device semi-angles at half power, and are given by $m_{i, t}=-\ln 2 / \ln \left(\cos \left(\phi_{i, 1 / 2}\right)\right)$ and $m_{i j, r}=$ $-\ln 2 / \ln \left(\cos \left(\psi_{i j, 1 / 2}\right)\right)$, respectively. The second step of (5) is obtained by substituting (4) in the first step and the constants of (4) are absorbed in $C_{j, R F} . \phi_{i j}$ and $\psi_{i j}$ in (6) and (7) are determined by the location of the device and the PD direction at the device, which are available after each realization of the system. Then, $G_{t}^{2}$ and $G_{r}^{2}$ can be easily obtained by using (6) and (7), respectively.

In practical scenarios, after each realization of the deployment of the system, each device is able to adjust its PDs direction $\theta_{R}=\arccos \left(\frac{H_{j}}{d_{j}}\right)$ (where $H_{j}$ is the height of the $j$ th device from the LED lamp in the vertical direction) degree from the horizontal direction to promise the incidence line is perpendicular to the PD axis. Then, in the following, we assume the incidence line is perpendicular to the PD axis at each device to maximize the received light. Then, it deduces $\psi_{i j}=0$ for $1 \leq i \leq L$. It is also assumed that $d_{i j}=d_{j}$ and $\phi_{i j}=\phi_{j}$ to simplify the analysis, as all LED lamps are close to each other and the area of the PD is normally on the order of $1 \mathrm{~cm}^{2}$. Therefore, (5) can be rewritten as

$$
\begin{aligned}
P_{i j, R F} & =\frac{C_{j, R F} P_{t}^{2} \Delta_{0}}{d_{j}^{4}} \cos ^{2 m_{i, t}}\left(\phi_{i j}\right) \cos ^{2 m_{i j, r}}\left(\psi_{i j}\right) \\
& =\frac{P_{t}^{2}}{d_{j}^{4}} \mathrm{H}_{i j},
\end{aligned}
$$

where $\Delta_{0}=\cos ^{2 m_{i j, r}}\left(\psi_{\Delta}\right)(0<\Delta \leq 1)$ is the orientation error of the PD, which is due to the estimated error of $\psi_{i j}$ $\left(\psi_{\Delta}, 0 \leq \psi_{\Delta}<\psi_{i j}\right)$, when the positional information of the $j$ th device is imperfect, and $\mathrm{H}_{i j}=C_{j, R F} \Delta_{0} \cos ^{2 m_{i, t}}\left(\phi_{j}\right)$.

\section{B. Light EH Model}

In this subsection, we will introduce the light EH model proposed in $[17,18]$.

In this work, we denote the link from the $i$ th lamp to the $j$ th device as downlink, the one from the $j$ th device to the RF receiver as uplink. We also denote the time duration for the downlink between the $i$ th lamp and the $j$ th device as $T_{j, d n}$, and the one for the uplink from the $j$ th device to the RF receiver as $T_{j, u p}$. There is $\eta_{i j}\left(0 \leq \eta_{i j} \leq 1\right)$ portion of $T_{j, d n}$ used for the data transmission from the $i$ th lamp to the $j$ th device, and the other $1-\eta_{i j}$ portion of $T_{j, d n}$ used for light $\mathrm{EH}$.

As in indoor energy constrained systems (e.g., wireless sensor systems) the traffic load over the downlink is very small (mainly including some control signaling to set up the data transmission), resulting short time duration for the data transmission over the downlink. Moreover, by considering the PD based light EH model proposed in [15], both of the AC and DC components of the received light signal can be harvested. Then, considering the characteristic of the traffic load over the downlink, it is reasonable to consider a time splitting (TS) based light energy harvesting model in this work, as shown in Fig. 2, to fully make use of the light signal in the idle state of the downlink and to avoid the circuit module for splitting the AC and DC components of the received light signal.

As suggested by [32] and [33], in a carrier allocation-VLC system, various RF carriers are adopted for signal modulation. Thus, the receiver can discriminate the signals transmitted from multiple LED lamps after photoelectric conversion, by using bandpass filters. Then, the signals from the other lights can be adopted for energy harvesting when the $i$ th light is chosen for the information delivery at the $j$ th device.

It is assumed that only one LED light is chosen for the data transmission over the downlink (e.g., the $i$ th light is selected for data transmission to the $j$ th device). Then, under TS scheme, the light from the $i$ th light will only be used for information decoding during the data transmission stage, while the light signal from the $i$ th light will be adopted for EH in light EH stage, as shown in Fig. 2.

Therefore, during the light EH stage over the VLC downlink which presented by the green part in Fig. 2, the device can harvest energy from the VLC signal transmitted by all $L$ ELD lamps. Then, at the $j$ th device, the energy harvested in the light EH stage can be written as [18]

$$
E_{j, L E H}=0.75 T_{i j, d n} V_{t} I_{D C}^{2} L^{2} \rho_{j}\left(1-\eta_{i j}\right) / I_{0},
$$

where $V_{t}$ is the thermal voltage $\left(V_{t} \approx 25 \mathrm{mV}\right), \rho_{j}$ is the $\mathrm{EH}$ efficiency at the $j$ th device ${ }^{4}, I_{0}$ is the dark saturation current of the photo-detector, $I_{D C}$ is the DC component of the output current from the photo-detector, which can be written as

$$
I_{D C}=\xi h_{i j} P_{t} B,
$$

where $\xi$ is the photo-detector responsivity in $\mathrm{A} / \mathrm{W}, B$ is the DC bias added to the electrical modulated signal to insure that the resulting signal is non-negative, before being used to modulate the optical intensity of a LED array.

Then, inserting (2) and (10) into (9) and considering that $I_{0}$ is on the order of $10^{-9} \sim 10^{-12}$ Ampere, one can obtain

\footnotetext{
${ }^{4}$ In this work we treat the EH efficiency as a constant. Because the received light power at each device should range from the reverse breakdown voltage to the turn-on voltage when the PD is used as a rectifier [34]. Then, a linear relationship is held between the received light power and the output current at the PD, which indicates that the EH efficiency is a constant.
} 
$E_{j, L E H}$ is upper bounded by [18]

$$
E_{j, L E H}=\Xi P_{t}^{2} T_{i j, d n} L^{2} \rho_{j}\left(1-\eta_{i j}\right) \frac{\cos ^{2 m_{i, t}}\left(\phi_{j}\right)}{d_{j}^{4}},
$$

where $\Xi=\frac{0.75 \Delta_{0} V_{t} \xi^{2} B^{2}\left(m_{i, t}+1\right)^{2} A^{2}\left(T_{s}\left(\psi_{\Delta}\right) g\left(\psi_{\Delta}\right)\right)^{2}}{4 \pi^{2} I_{0}}$.

Moreover, the devices are able to harvest energy from the light signals transmitted by LED lamps, no matter these light signals are used for delivering energy or information bits. In other words, the light from the other LED lamps, which is adopted for information delivery, can also be used for light EH. Then, the light from the other lamps are used for EH in both light EH and data transmission stages. So, when in the data transmission stage over the VLC link which is depicted by the pink part in Fig. 2, the $j$ th device can harvest energy from all LED lamps except for the $i$ th LED lamp. Thus, when data is transmitted from the $i$ th LED lamp to the $j$ th device over the VLC downlink, we can give the energy harvested from the other lamps as $E_{j, D T}=\Xi P_{t}^{2}(L-1)^{2} T_{i j, d n} \rho_{j} \eta_{i j} \frac{\cos ^{2 m_{i, t}}\left(\phi_{j}\right)}{d_{j}^{4}}$.

In this paper, it is assumed that all battery capacities are large enough to simplify the analysis. In other words, the harvested energy will not exceed the battery capacity at each device. Then, considering all LED lamps are identical, the harvested energy at the $j$ th device can be given as

$$
\begin{aligned}
E_{j}= & E_{j, L E H}+E_{j, D T} \\
= & \frac{P_{t}^{2} \Xi T_{i j, d n} \rho_{j}}{d_{j}^{4}}\left[L^{2}\left(1-\eta_{i j}\right) \cos ^{2 m_{i, t}}\left(\phi_{j}\right)\right. \\
& \left.\quad+\eta_{i j}(L-1)^{2} \cos ^{2 m_{i, t}}\left(\phi_{j}\right)\right] \\
= & P_{t}^{2} \Xi T_{i j, d n} \rho_{j}\left(L^{2}-2 L \eta_{i j}+\eta_{i j}\right) \frac{\cos ^{2 m_{i, t}}\left(\phi_{j}\right)}{d_{j}^{4}} .
\end{aligned}
$$

In the considered system, one can see that the transmit power over the RF uplink is decided by the harvested energy because of the following two folds: 1) Due to the battery size (normally on the order of $500 \mathrm{mAh}$ ) and the low power conversion efficiency of the PD (normally about $12 \%$ ), it is reasonable to assume that the battery size is too large for light EH to fully change. 2) In the indoor energy constrained systems, normally the devices, e.g., wireless sensor nodes, work in low duty cycle and have very limited data to deliver to the RF receiver. Therefore, the duration of the data transmission over the RF uplink will not be long.

In this work, we also assume that all the energy adopted for the information delivery over the RF uplink comes from the harvested one over the VLC downlink. Therefore, the average transmit power at the $j$ th device over the uplink can be expressed as

$$
\begin{aligned}
P_{j} & =\frac{\zeta E_{j}}{T_{j, u p}} \\
& =\frac{\zeta P_{t}^{2} \Xi T_{i j, d n} \rho_{j}\left(L^{2}-2 L \eta_{i j}+\eta_{i j}\right)}{T_{j, u p}} \cdot \frac{\cos ^{2 m_{i, t}}\left(\phi_{j}\right)}{d_{j}^{4}} \\
& =\Psi_{i j} \frac{\cos ^{2 m_{i, t}}\left(\phi_{j}\right)}{d_{j}^{4}}
\end{aligned}
$$

where $\zeta$ is the utilization factor of the harvested energy for the data transmission over the RF uplink, and $\Psi_{i j}=$ $\frac{\zeta P_{t}^{2} \Xi T_{i j, d n} \rho_{j}\left(L^{2}-2 L \eta_{i j}+\eta_{i j}\right)}{T_{j, u p}}$.

As VLC and RF are orthogonal to each other, the data transmission over the VLC downlink and RF uplink can occur simultaneously. However, under this case, it is also reasonable to assume that the harvested energy, which is harvested before the data transmission over the RF uplink starting, is fully used for the data transmission over RF link. Thus, the proposed TS based light EH model presented in Fig. 3 and (10) still holds for this case.

\section{Outage Analysis For the VLC Downlink}

In this section, we will study the outage performance of the VLC downlink, which has nothing to do with light energy harvesting due to the adopted TS based light EH scheme.

Using (5), we can easily obtain the received SNR of the VLC signal from the $i$ th lamp to the $j$ th device as

$$
\begin{aligned}
\gamma_{i j, R F} & =\frac{P_{i j, R F}}{N_{0} d_{j}^{4}} \\
& =\frac{P_{t}^{2} C_{j, R F} \Delta_{0}}{N_{0}} \cdot \frac{\cos ^{2 m_{i, t}}\left(\phi_{j}\right)}{d_{j}^{4}},
\end{aligned}
$$

where $N_{0}$ is the average power of the complex Gaussian noise at all devices.

To promise the information transmission between the lamp and the devices, the received SNR at the device should be larger than a predefined threshold, $\gamma_{0}$; otherwise, outage event will occur as the device can not successfully decode the information transmitted by the lamp.

Therefore, the OP for the VLC downlink, $\operatorname{Pr}_{\mathrm{VLC}}$, can be given as

$$
\begin{aligned}
\operatorname{Pr}_{\mathrm{VLC}} & =\operatorname{Pr}\left\{\gamma_{i j} \leq \gamma_{0}\right\} \\
& =\operatorname{Pr}\left\{\frac{P_{t}^{2} C_{j, R F} \Delta_{0}}{N_{0}} \cdot \frac{\cos ^{2 m_{i, t}}\left(\phi_{j}\right)}{d_{j}^{4}} \leq \gamma_{0}\right\} .
\end{aligned}
$$

Theorem 1. Considering the randomness of the distribution of the device and using (1) and spherical coordinates, we have

$$
\operatorname{Pr}\left\{d_{i}^{p} \leq a \cos ^{q}\left(\phi_{i}\right)\right\}=\left\{\begin{array}{ll}
1-\frac{3 q}{3 q+p} a^{-\frac{1}{q}} D^{\frac{p}{q}}, & \text { if } a^{\frac{1}{p}} \geq D \\
\frac{p}{3 q+p} \frac{1}{D^{3}} a^{\frac{3}{p}}, & \text { else }
\end{array},\right.
$$

where $i=1,2, a, p$ and $q$ are positive constants.

Proof: Please refer to Part A of the Appendix.

Considering the randomness of the distribution of the device and using (16), $\operatorname{Pr}_{\mathrm{VLC}}$ can be easily calculated as

$$
\operatorname{Pr}_{\mathrm{VLC}}=\left\{\begin{array}{ll}
\frac{3 q_{V}}{3 q_{V}+p_{V}} a_{V}^{-\frac{1}{q_{V}}} D^{\frac{p_{V}}{q_{V}}}, & \text { if } a_{V}^{\frac{1}{p_{V}}} \geq D \\
1-\frac{p_{V}}{3 q_{V}+p_{V}} \frac{1}{D^{3}} a_{V}^{\frac{3}{p_{V}}}, & \text { else }
\end{array},\right.
$$

where $a_{V}=\frac{P_{t}^{2} C_{j, R F} \Delta_{0}}{\gamma_{0} N_{0}}, p_{V}=4$ and $q_{V}=2 m_{i, t}$.

Remark 1. The OP over the VLC downlink is related to the transmission parameters at the LED lamp and the receiving parameters at the devices, while light EH at the device is irrelevant to the outage performance over the VLC downlink. 


\section{OUtAge ANALYsis FOR THE RF UPLINK}

In indoor scenarios, there may be multiple devices of a same type, e.g., indoor air quality/temperature/moisture sensors, which report the monitoring data at the same time. In this case, we assume NOMA scheme is adopted to deal with the concurrent transmissions over the uplink. We denote this case as interference scenario.

\section{A. Signal Model for NOMA over the Uplink}

In this case, there are $N(N>1)$ devices forward their data to the RF receiver. Then, NOMA scheme is adopted to allow all devices to make use of the frequency resource simultaneously.

In this work, we assume that the channels among the devices and the RF receiver follow Rician distribution. Then, the PDF and CDF of the power gain of the link between the $j$ th device and the RF receiver, $\left|h_{j}\right|^{2}$, can be given as

$$
\begin{aligned}
f_{\left|h_{j}\right|^{2}}(x)= & \frac{(1+K)}{\Omega} \cdot \exp \left(-K-\frac{x(1+K)}{\Omega}\right) \\
& \cdot I_{0}\left(2 \sqrt{\frac{K(1+K) x}{\Omega}}\right), x \geq 0
\end{aligned}
$$

and

$$
F_{\left|h_{j}\right|^{2}}(x)=1-Q_{1}\left(\sqrt{2 K}, \sqrt{\frac{2(1+K) x}{\Omega}}\right),
$$

respectively, where $\Omega$ is the variance of the signal, $K$ is Rician factor which corresponds to the ratio of the power of the LOS (specular) component to the average power of the scattered component, $Q_{1}(a, b)=\int_{b}^{\infty} x \cdot \exp \left(-\frac{x^{2}+a^{2}}{2}\right) I_{0}(a x) d x$ is Marcum- $Q$ function, $I_{0}(\cdot)$ is modified Bessel function of order 0 .

The received signal at the $\mathrm{RF}$ receiver can be written as

$$
y_{R F}=\sum_{j=1}^{N} \sqrt{\frac{P_{j, N O M A}}{d_{j}{ }^{n}}} h_{j} x_{j}+z_{R F},
$$

where $x_{j}$ is the transmitted data from the $j$ th device, $h_{j}$ is the channel gain from the $j$ th device to the RF receiver, $z_{R F}$ is the AWGN at the RF receiver with average power $N_{0}, n$ is the path loss exponent, the transmit power at the $j$ th device $P_{j, N O M A}$ can be given as

$$
P_{j, N O M A}=\min \left\{P_{j}, P_{t g} d_{j}{ }^{n}\right\},
$$

where $P_{j}=\frac{\Psi_{i j}}{d_{j}^{4}} \cos ^{2 m_{i, t}}\left(\phi_{j}\right)$ given by (13) in Section III, $P_{t g}$ is the target average received power at the RF receiver.

As NOMA is adopted over the uplinks, successive interference cancelation (SIC) receiver requires that all the received power from each device can be distinguished [37]. Therefore, the transmit power at the $j$ th and $k$ th $(1 \leq k<j \leq N)$ devices should satisfy the following rule:

$$
\frac{P_{k, N O M A}}{P_{j, N O M A}}=\frac{d_{k}{ }^{n}}{d_{j}{ }^{n}} \times 10^{(j-k) \Delta},
$$

where $\Delta>0$ is the power back-off step of the target received power.
Then, $P_{j, N O M A}$ can be rewritten as

$$
\begin{aligned}
P_{j, N O M A} & =\min \left\{P_{j}, \frac{P_{t g} d_{j}{ }^{n}}{10^{(j-1) \Delta}}\right\} \\
& =\min \left\{\frac{\Psi_{i j}}{d_{j}^{4}} \cos ^{2 m_{i, t}}\left(\phi_{j}\right), \frac{P_{t g} d_{j}{ }^{n}}{10^{(j-1) \Delta}}\right\} .
\end{aligned}
$$

Under NOMA scheme, before decoding the $j$ th signal, the RF receiver can detect the prior $l$ th $(l<j)$ signal by using SIC. Then, the rest $(N-j)$ devices' signals will be regarded as interferences during decoding the $j$ th signal ${ }^{5}$. Therefore, the received signal-to-interference-plus-noise ratio (SINR) of the $j$ th device can be given as

$$
\gamma_{j}=\left\{\begin{array}{ll}
\frac{P_{j, N O M A}\left|h_{j}\right|^{2} / d_{j}^{n}}{\sum_{l=j+1}^{N} P_{l, N O M A}\left|h_{j}\right|^{2} / d_{j}^{n}+N_{0}}, & \text { if } j<N \\
\frac{P_{M, N O M A}\left|h_{N}\right|^{2} / d_{N}^{n}}{N_{0}}, & \text { if } j=N
\end{array} .\right.
$$

On one hand, observing (24), one can easily find that, when $N>2$, it is hard to obtain a closed-form expression for the PDF of $\gamma_{j}(j<N)$. However, it is possible to achieve the one while $N=2$, as given by [37]. On the other hand, when there are two devices, SIC can be carried on at the RF receiver by implementing suitable user pairing (in other words, selecting two devices to meet power disparity requirement for NOMA); On the contrary, there are more than two devices working under NOMA scheme, the implementation cost of SIC receiver, e.g., computational resources and the complexity of user paring algorithm, will unavoidably increase, which will undermine the performance of the system. User pairing is another topic of implementing NOMA, which is beyond the scope of this paper, the readers interested in this topic can refer to [38] and [39]. Therefore, in the following, we assume there are two devices being paired to work under NOMA scheme, which are uniformly distributed in the 3-D room.

Then, in this case, $\gamma_{j}$ can be rewritten as

$$
\gamma_{j}=\left\{\begin{array}{ll}
\frac{P_{1, N O M A}\left|h_{1}\right|^{2} / d_{1}^{n}}{P_{2, N O M A}\left|h_{1}\right|^{2} / d_{1}^{n}+N_{0}}, & \text { if } j=1 \\
\frac{P_{2, N O M A}\left|h_{2}\right|^{2} / d_{2}^{n}}{N_{0}}, & \text { if } j=2
\end{array} .\right.
$$

\section{B. Outage Analysis for NOMA}

As indicated in [40], the average noise power over the entire room area for a LED receiver with $70 \mathrm{MHz}$ bandwidth is $-98.82 \mathrm{dBm}$. The noise power (mainly including the channel and antenna noise) for $22 \mathrm{MHz} 802.11 \mathrm{~b}$ or $20 \mathrm{MHz} 802.11 \mathrm{~g}$ is about $-100 \mathrm{dBm}$ at room temperature [41] and the noise density of at the sensor receiver is $10^{-16}$ and $10^{-17} \mathrm{~V}^{2} / \mathrm{Hz}$ at $1 \mathrm{MHz}$ and $1000 \mathrm{MHz}$, respectively, as shown by Fig. 3.6 in [42]. The transmit power of wireless devices (e.g., WiFi devices and wireless sensors) are normally on the order of

\footnotetext{
${ }^{5}$ In this work, we assume that the order of the $l$ th device is prior to the $j$ th device, namely, $P_{l, N O M A}\left|h_{l}\right|^{2} / d_{l}^{n} \geq P_{j, N O M A}\left|h_{j}\right|^{2} / d_{j}^{n}$. Moreover, normally, the RF receiver suffers two kinds of interferences: in-band and out-band interferences. As out-band interference can be easily detected and removed by using band-pass filters and NOMA systems are self-interference systems, in this work we mainly focus on the effect of in-band interference on the performance of the target systems.
} 
tens of $\mathrm{mW}$ [40]- [42]. Thus, we can see that the received power in indoor scenarios is normally on the order of $\mathrm{mW}$, as the transmission distance is on the order of meters and the pathloss factor in indoor scenarios ranges from 1 to 3 . Then, we can see that the noise power is quite small compared to the received signal power. Thus, in this work it is reasonable for us to ignore the noise in the received signal at device 1 for simplification.

Considering NOMA, the OP for the two devices can be expressed as

$$
\begin{aligned}
\operatorname{Pr}_{1} & =\operatorname{Pr}\left\{\gamma_{1} \leq \gamma_{t h}\right\} \\
& =\operatorname{Pr}\left\{\frac{P_{1, N O M A}\left|h_{1}\right|^{2} / d_{1}^{n}}{P_{2, N O M A}\left|h_{1}\right|^{2} / d_{1}^{n}+N_{0}} \leq \gamma_{t h}\right\} \\
& \approx \operatorname{Pr}\left\{P_{1, N O M A} \leq P_{2, N O M A} \gamma_{t h}\right\}
\end{aligned}
$$

and

$$
\begin{aligned}
\operatorname{Pr}_{2} & =\operatorname{Pr}\left\{\gamma_{2} \leq \gamma_{t h}\right\} \\
& =\operatorname{Pr}\left\{\frac{P_{2, N O M A}\left|h_{2}\right|^{2}}{d_{2}^{n} N_{0}} \leq \gamma_{t h}\right\},
\end{aligned}
$$

respectively.

When $\frac{\Psi_{i j}}{d_{j}^{4}} \cos ^{2 m_{i, t}}\left(\phi_{j}\right) \geq \frac{P_{t g} d_{j}{ }^{n}}{10^{(j-1) \Delta}}$ and $j=1, P_{1}=P_{t g} d_{1}{ }^{n}$ and $P_{2}=\min \left\{\frac{P_{t g} d_{2}{ }^{n}}{10^{\Delta}}, \frac{\Psi_{i 2}}{d_{2}^{4}} \cos ^{2 m_{i, t}}\left(\phi_{2}\right)\right\}^{6}$. In this case, we can calculate the OP under two subcases according to the value of $P_{2, N O M A}$ as follows:

(1) When $\frac{P_{t g} d_{2}{ }^{n}}{10^{\Delta}} \leq \frac{\Psi_{i 2}}{d_{2}^{4}} \cos ^{2 m_{i, t}}\left(\phi_{2}\right), P_{2, N O M A}=\frac{P_{t g} d_{2}{ }^{n}}{10^{\Delta}}$.

Then, we can have

$$
\begin{aligned}
\operatorname{Pr}_{1} & \approx \operatorname{Pr}\left\{P_{t g} d_{1}{ }^{n} \leq \frac{P_{t g} \gamma_{t h}}{10^{\Delta}} d_{2}{ }^{n}\right\} \\
& =\operatorname{Pr}\left\{d_{2}{ }^{n} \geq \frac{10^{\Delta}}{\gamma_{t h}} d_{1}{ }^{n}\right\} \\
& =I_{1}
\end{aligned}
$$

and

$$
\begin{aligned}
\operatorname{Pr}_{2} & =\operatorname{Pr}\left\{\gamma_{2} \leq \gamma_{t h}\right\} \\
& =\operatorname{Pr}\left\{P_{t g}\left|h_{2}\right|^{2} \leq 10^{\Delta} N_{0} \gamma_{t h}\right\} \\
& =I_{2} .
\end{aligned}
$$

(2) When $\frac{P_{t g} d_{2}{ }^{n}}{10^{\Delta}}>\frac{\Psi_{i 2}}{d_{2}^{4}} \cos ^{2 m_{i, t}}\left(\phi_{2}\right), P_{2, \text { NOMA }}=$ $\frac{\Psi_{i 2}}{d_{2}^{4}} \cos ^{2 m_{i, t}}\left(\phi_{2}\right)$.

Then, we can obtain

$$
\begin{aligned}
\operatorname{Pr}_{1} & \approx \operatorname{Pr}\left\{d_{2}{ }^{4} \leq \frac{\Psi_{i 2} \gamma_{t h}}{P_{t g}} \frac{1}{d_{1}^{n}} \cos ^{2 m_{i, t}}\left(\phi_{2}\right)\right\} \\
& =I_{3}
\end{aligned}
$$

\footnotetext{
${ }^{6}$ In practice, there exists a case: $P_{t g} d_{2}{ }^{n} 10^{-\Delta} \geq P_{t g} d_{2}{ }^{n}$, where $P_{t g} d_{2}{ }^{n}$ is the transmit power for device 2 obtained from the second item in (21). However, the received power of the signal transmitted by device 2 at the RF receiver will be larger than $P_{t g}$, leading to the improved received SNR. Then, in this work we let the transmit power at device 2 is decided by (22) to avoid increasing interference to the received signal for device 1.
}

and

$$
\begin{aligned}
\operatorname{Pr}_{2} & =\operatorname{Pr}\left\{\gamma_{2} \leq \gamma_{t h}\right\} \\
& =\operatorname{Pr}\left\{\frac{\Psi_{i 2}}{d_{2}^{n+4}} \cos ^{2 m_{i, t}}\left(\phi_{2}\right)\left|h_{2}\right|^{2} \leq N_{0} \gamma_{t h}\right\} \\
& =I_{4} .
\end{aligned}
$$

When $\frac{\Psi_{i j}}{d_{j}^{4}} \cos ^{2 m_{i, t}}\left(\phi_{j}\right)<\frac{P_{t g} d_{j}{ }^{n}}{10^{(j-1) \Delta}}$ and $j=1$, $P_{1, \text { NOMA }}=\frac{\Psi_{i 1}}{d_{1}^{4}} \cos ^{2 m_{1, t}}\left(\phi_{1}\right)$ and $P_{2, \text { NOMA }}=$ $\min \left\{\frac{\Psi_{i 1}}{10^{\Delta}} \cdot \frac{d_{2}{ }^{n}}{d_{1}{ }^{n+4}} \cos ^{2 m_{i, t}}\left(\phi_{1}\right), \frac{\Psi_{i 2}}{d_{2}^{4}} \cos ^{2 m_{i, t}}\left(\phi_{2}\right)\right\}$.

Similarly, in this case we can also calculate the OP under two subcases according to the value of $P_{2, N O M A}$ as follows:

(1) When $\frac{\Psi_{i 1}}{10^{\Delta}} \cdot \frac{d_{2}{ }^{n}}{d_{1}{ }^{n+4}} \cos ^{2 m_{i, t}}\left(\phi_{1}\right) \leq \frac{\Psi_{i 2}}{d_{2}^{4}} \cos ^{2 m_{i, t}}\left(\phi_{2}\right)$, $P_{2, \text { NOMA }}=\frac{\Psi_{i 1}}{10^{\Delta}} \cdot \frac{d_{2}{ }^{n}}{d_{1}{ }^{n+4}} \cos ^{2 m_{i, t}}\left(\phi_{1}\right)$.

Then, we can achieve

$$
\begin{aligned}
\operatorname{Pr}_{1} & \approx \operatorname{Pr}\left\{\frac{\Psi_{i 1}}{d_{1}^{4}} \cos ^{2 m_{i, t}}\left(\phi_{1}\right) \leq \frac{\Psi_{i 1} \gamma_{t h}}{10^{\Delta}} \cdot \frac{d_{2}{ }^{n}}{d_{1}{ }^{n+4}} \cos ^{2 m_{i, t}}\left(\phi_{1}\right)\right\} \\
& =\operatorname{Pr}\left\{d_{1}{ }^{n} \leq \frac{\gamma_{t h}}{10^{\Delta}} \cdot d_{2}{ }^{n}\right\} \\
& =I_{1}
\end{aligned}
$$

and

$$
\begin{aligned}
\operatorname{Pr}_{2} & =\operatorname{Pr}\left\{\gamma_{2} \leq \gamma_{t h}\right\} \\
& =\operatorname{Pr}\left\{\frac{\Psi_{i 1}}{10^{\Delta}} \cdot \frac{1}{d_{1}^{n+4}} \cos ^{2 m_{i, t}}\left(\phi_{1}\right)\left|h_{2}\right|^{2} \leq N_{0} \gamma_{t h}\right\} \\
& =I_{5} .
\end{aligned}
$$

(2) When $\frac{\Psi_{i 1}}{10^{\Delta}} \cdot \frac{d_{2}^{n}}{d_{1}^{n+1}} \cos ^{2 m_{i, t}}\left(\phi_{1}\right)>\frac{\Psi_{i 2}}{d_{2}^{4}} \cos ^{2 m_{i, t}}\left(\phi_{2}\right)$, $P_{2, \text { NOMA }}=\frac{\Psi_{i 2}}{d_{2}^{4}} \cos ^{2 m_{i, t}}\left(\phi_{2}\right)$.

Then, we can have

$$
\begin{aligned}
\operatorname{Pr}_{1} & \approx \operatorname{Pr}\left\{\frac{\Psi_{i 1}}{d_{1}^{4}} \cos ^{2 m_{i, t}}\left(\phi_{1}\right) \leq \frac{\Psi_{i 2}}{d_{2}^{4}} \cos ^{m_{i, t}}\left(\phi_{2}\right) \gamma_{t h}\right\} \\
& =I_{6}
\end{aligned}
$$

and

$$
\begin{aligned}
\operatorname{Pr}_{2} & =\operatorname{Pr}\left\{\gamma_{2} \leq \gamma_{t h}\right\} \\
& =\operatorname{Pr}\left\{\frac{\Psi_{i 2}}{d_{2}^{n+4}} \cos ^{2 m_{i, t}}\left(\phi_{2}\right)\left|h_{2}\right|^{2} \leq N_{0} \gamma_{t h}\right\} \\
& =I_{4} .
\end{aligned}
$$

Therefore, we can finally obtain

$$
\begin{aligned}
\operatorname{Pr}_{1, \text { NOMA }}= & I_{P_{1}} \cdot\left[I_{P_{21}} \cdot I_{1}+\left(1-I_{P_{21}}\right) \cdot I_{3}\right] \\
& +\left(1-I_{P_{1}}\right) \cdot\left[I_{P_{22}} \cdot I_{1}+\left(1-I_{P_{22}}\right) \cdot I_{6}\right]
\end{aligned}
$$

and

$$
\begin{aligned}
\operatorname{Pr}_{2, N O M A}= & I_{P_{1}} \cdot\left[I_{P_{21}} \cdot I_{2}+\left(1-I_{P_{21}}\right) \cdot I_{4}\right]+\left(1-I_{P_{1}}\right) \\
& \cdot\left[I_{P_{22}} \cdot I_{5}+\left(1-I_{P_{22}}\right) \cdot I_{4}\right],
\end{aligned}
$$

where $I_{P_{1}}=\operatorname{Pr}\left\{\frac{\Psi_{i 1}}{d_{1}^{4}} \cos ^{2 m_{i, t}}\left(\phi_{1}\right) \geq P_{t g} d_{1}{ }^{n}\right\}$, $I_{P_{21}}=\operatorname{Pr}\left\{\frac{P_{t g} d_{2}{ }^{n}}{10^{\Delta}} \leq \frac{\Psi_{i 2}}{d_{2}^{4}} \cos ^{2 m_{i, t}}\left(\phi_{2}\right)\right\} \quad$ and $I_{P_{22}}=\operatorname{Pr}\left\{\frac{\Psi_{i 1}}{10^{\Delta}} \cdot \frac{d_{2}^{n}}{d_{1}^{n+4}} \cos ^{2 m_{i, t}}\left(\phi_{1}\right) \leq \frac{\Psi_{i 2}}{d_{2}^{4}} \cos ^{2 m_{i, t}}\left(\phi_{2}\right)\right\}$. 
In the following, we will calculate the items in (36) and (37), respectively.

Recalling (16) presented in last section, we can easily derive $I_{P_{1}}$ and $I_{P_{21}}$ as

$I_{P_{1}}=\left\{\begin{array}{ll}1-\mathrm{X}_{1}{ }^{-\frac{n+4}{2 m_{i, t}}} \frac{6 m_{i, t}}{6 m_{i, t}+n+4} D^{\frac{n+4}{2 m_{i, t}}}, & \text { if } \mathrm{X}_{1}>D^{\frac{1}{n+4}} \\ \frac{1}{D^{3}} \mathrm{X}_{1}^{3} \frac{n+4}{n+4+6 m_{i, t}}, & \text { else }\end{array}\right.$,

and

$I_{P_{21}}= \begin{cases}1-\mathrm{X}_{2}{ }^{-\frac{n+4}{2 m_{i, t}}} \frac{6 m_{i, t}}{6 m_{i, t}+n+4} D^{\frac{n+4}{2 m_{i, t}}}, & \text { if } \mathrm{X}_{2}>D^{\frac{1}{n+4}} \\ \frac{1}{D^{3}} \mathrm{X}_{2}{ }^{3} \frac{n+4}{n+4+6 m_{i, t}}, & \text { else }\end{cases}$

respectively, where $\mathrm{X}_{1}=\left(\frac{\Psi_{i 1}}{P_{t g}}\right)^{\frac{1}{n+4}}$ and $\mathrm{X}_{2}=\left(\frac{\Psi_{i 2} 10^{\Delta}}{P_{t g}}\right)^{\frac{1}{n+4}}$.

Similar to the derivation of Theorem 1 , one can easily have

$$
I_{1}=\left\{\begin{array}{ll}
1-\frac{1}{2} \mathrm{X}_{3}^{-\frac{3}{n}}, & \text { if } \mathrm{X}_{3} \geq 1 \\
\frac{1}{2} \mathrm{X}_{3}^{\frac{3}{n}}, & \text { else }
\end{array},\right.
$$

where $\mathrm{X}_{3}=\gamma_{t h} / 10^{\Delta}$.

To calculate $I_{P_{22}}$ and $I_{6}$, we prove a theorem as follows:

Theorem 2. Considering the randomness of the distribution of the device and using (1) and spherical coordinates, we can calculate $T=\operatorname{Pr}\left\{d_{2} \leq a d_{1} \frac{\cos ^{p}\left(\phi_{2}\right)}{\cos ^{q}\left(\phi_{1}\right)}\right\}$ (where $a, p$ and $q$ are positive constants) as

$T=\left\{\begin{array}{ll}1-\frac{3 p \mathrm{~B}}{1+3 p} a^{-\frac{1}{p}}-\frac{\mathrm{C}}{2} a^{-3}, & \text { if } a>1 \\ \left(1-\frac{\mathrm{C}}{2}-\frac{3 p \mathrm{~B}}{1+3 p}-\frac{3 q \mathrm{~A}}{3 q-1}\right) a^{3}+\frac{3 q \mathrm{~A}}{3 q-1} a^{\frac{1}{q}}, & \text { else }\end{array}\right.$,

where $\mathrm{A}=\frac{3 q}{1+3 q} \frac{q}{p+q}, \mathrm{~B}=1-\mathrm{A}-\mathrm{C}$ and $\mathrm{C}=\frac{1}{1+3 q} \frac{1}{1-3 p}$.

Proof: Please refer to Part $B$ of the Appendix.

Then, by employing Theorem 2, we can easily derive $I_{P_{22}}$ and $I_{6}$ as

$I_{P_{22}}=\left\{\begin{array}{l}1-\frac{3 p_{1} \mathrm{~B}_{1}}{1+3 p_{1}} a_{1}^{-\frac{1}{p_{1}}}-\frac{\mathrm{C}_{1}}{2} a_{1}^{-3} \\ \left(1-\frac{\mathrm{C}_{1}}{2}-\frac{3 p_{1} \mathrm{~B}_{1}}{1+3 p_{1}}-\frac{3 q_{1} \mathrm{~A}_{1}}{3 q_{1}-1}\right) a_{1}^{3}+\frac{3 q_{1} \mathrm{~A}_{1}}{3 q_{1}-1} a_{1}^{\frac{1}{q_{1}}},\end{array}\right.$

if $a>1_{I_{5}}=3 \sum_{k=0}^{\infty}(-1)^{k} \exp (-K) \frac{L_{k}^{(0)}(K)}{\Gamma(k+2)}\left(\frac{10^{\Delta} \gamma_{t h} N_{0}(1+K)}{\Omega \Psi_{i 1}}\right)^{k+1}$

and

$$
I_{6}= \begin{cases}1-\frac{3 p_{2} \mathrm{~B}_{2}}{1+3 p_{2}} a_{2}^{-\frac{1}{p_{2}}}-\frac{\mathrm{C}_{2}}{2} a_{2}^{-3}, & \text { if } a> \\ \left(1-\frac{\mathrm{C}_{2}}{2}-\frac{3 p_{2} \mathrm{~B}_{2}}{1+3 p_{2}}-\frac{3 q_{2} \mathrm{~A}_{2}}{3 q_{2}-1}\right) a_{2}^{3}+\frac{3 q_{2} \mathrm{~A}_{2}}{3 q_{2}-1} a_{2}^{\frac{1}{q_{2}}}, & \text { else }\end{cases}
$$

respectively, where $\mathrm{A}_{i}=\frac{3 q_{i}}{1+3 q_{i}} \frac{q_{i}}{p_{i}+q_{i}}, \mathrm{~B}_{i}=1-\mathrm{A}_{i}-\mathrm{C}_{i}$ and $\mathrm{C}_{i}=\frac{1}{1+3 q_{i}} \frac{1}{1-3 p_{i}}(i=1,2) ; a_{1}=\left(\frac{10^{\Delta} \Psi_{i 2}}{\Psi_{i 1}}\right)^{\frac{1}{n+4}}, p_{1}=\frac{2 m_{i, t}}{n+4}$, $q_{1}=\frac{2 m_{i, t}}{n+4} ; a_{2}=\left(\frac{\Psi_{i 2} \gamma_{t h}}{\Psi_{i 1}}\right)^{\frac{1}{4}}, p_{2}=\frac{m_{i, t}}{2}$ and $q_{2}=\frac{m_{i, t}}{2}$.

Using the similar method with the derivation of Theorem 2 , we can also derive $I_{3}$ as

$$
I_{3}=\left\{\begin{array}{ll}
1-\mathrm{C}_{3} D^{\frac{2}{m_{i, t}}}\left(\frac{D}{\mathrm{X}_{4}}\right)^{\frac{n}{2 m_{i, t}}}, & \text { if } \mathrm{X}_{4}>D^{1+\frac{4}{n}} \\
\mathrm{~A}_{3} \frac{\mathrm{X}_{4}{ }^{3}}{D^{3+\frac{12}{n}}}+\mathrm{B}_{3} \frac{\mathrm{X}_{4} \frac{3 n}{4}}{D^{3+\frac{3 n}{4}}}, & \text { else }
\end{array},\right.
$$

where $\mathrm{X}_{4}=\left(\frac{\Psi_{i 2} \gamma_{t h}}{P_{t g}}\right)^{\frac{1}{n}}, \mathrm{~A}_{3}=1+\mathrm{B}_{3}-\mathrm{C}_{3}, \mathrm{~B}_{3}=$ $\frac{6}{2+3 m_{i, t}} \frac{4}{12-3 n}$ and $\mathrm{C}_{3}=\frac{3 m_{i, t}}{2+3 m_{i, t}} \frac{6 m_{i, t}}{6 m_{i, t}+n}$.

Using (18) and (19), we easily obtain

$$
\begin{aligned}
I_{2} & =\operatorname{Pr}\left\{\left|h_{2}\right|^{2} \leq \frac{10^{\Delta} N_{0} \gamma_{t h}}{P_{t g}}\right\} \\
& =1-Q_{1}\left(\sqrt{2 K}, \sqrt{\frac{210^{\Delta} N_{0} \gamma_{t h}(1+K)}{P_{t g} \Omega}}\right) .
\end{aligned}
$$

To facilitate calculating the other items in (36) and (37), we present an useful theorem as follows:

Theorem 3. For $a>0, b \geq 0, p \geq 0$ and $q \geq 0$, it holds

$$
\begin{aligned}
\operatorname{Pr} & \left\{\frac{a}{d^{q}} \cos ^{p}(\phi)|h|^{2} \leq b\right\}=3 \sum_{k=0}^{\infty}(-1)^{k} \exp (-K) \\
\cdot & \frac{L_{k}^{(0)}(K)}{\Gamma(k+2)}\left(\frac{b(1+K)}{a \Omega}\right)^{k+1} \frac{D^{q(k+1)}}{q(k+1)+3} \cdot \frac{1}{1-p(k+1)},
\end{aligned}
$$

where $(d, \theta, \phi)$ is the spherical coordinate of a device which is uniformly distributed in our considered space, $h$ is the fading coefficient over Rician fading channels, $L_{k}^{(0)}(x)=$ $\sum_{l=0}^{k} \frac{k !}{(k-l) !(l !)^{2}}(-x)^{l}$ is the generalized Laguerre polynomial with degree $k$ and order 0, given by Eq. (22.3.9) in [44].

Proof: The proof of Theorem 3 can be found in Part $C$ of the Appendix.

Making use of Theorem 3, we can easily obtain

$$
\begin{aligned}
I_{4}= & 3 \sum_{k=0}^{\infty}(-1)^{k} \exp (-K) \frac{L_{k}^{(0)}(K)}{\Gamma(k+2)}\left(\frac{\gamma_{t h} N_{0}(1+K)}{\Omega \Psi_{i 2}}\right)^{k+1} \\
& \cdot \frac{D^{(n+4)(k+1)}}{3+(n+4)(k+1)} \cdot \frac{1}{1-2 m_{i, t}(k+1)}
\end{aligned}
$$

and

$$
\cdot \frac{D^{(n+4)(k+1)}}{3+(n+4)(k+1)} \cdot \frac{1}{1-2 m_{i, t}(k+1)} \text {. }
$$

Therefore, the OP for the two devices can be achieved by submitting (38), (39), (42), (40), (44) and (43) into (36), and (38), (39), (42), (45), (47) and (48) into (37), respectively.

\section{NUMERICAL RESULTS}

In this section, we first run Monte Carlo simulations to validate our analytical expressions of the OP for three different cases. Unless otherwise explicitly specified, the main parameters are set as $D=3 \mathrm{~m}, L=8, \xi=4 \mathrm{~A} / \mathrm{W}, I_{0}=10^{-9} \mathrm{~A}$, $V_{t}=25 \mathrm{mV}, A=4 \times 10^{-4} \mathrm{~m}^{2}, B=5 \mathrm{~mA}, \rho=0.8, \eta=0.1$, $\zeta=1, n=2, T_{d n}=10^{7} \mathrm{~s}, K=2, M=2, T_{u p} / T_{d n}=0.01$, $\phi_{1 / 2}=85^{\circ}, \gamma_{0}=\gamma_{t h}=0 \mathrm{~dB}, \Delta=0.001, C_{R F}=0.1$, $N_{0, j}=1$. In order to model the randomness of the position of the devices, $5 \times 10^{4}$ times of the realizations of the considered system have been carried on in the following part. Moreover, 
we let the device transmits $10^{5}$ bits to the RF receiver on the roof to address the ergodic characters of Rician fading, and there are 50 bits transmitted over the VLC downlink in the second stage presented in Fig. 2.

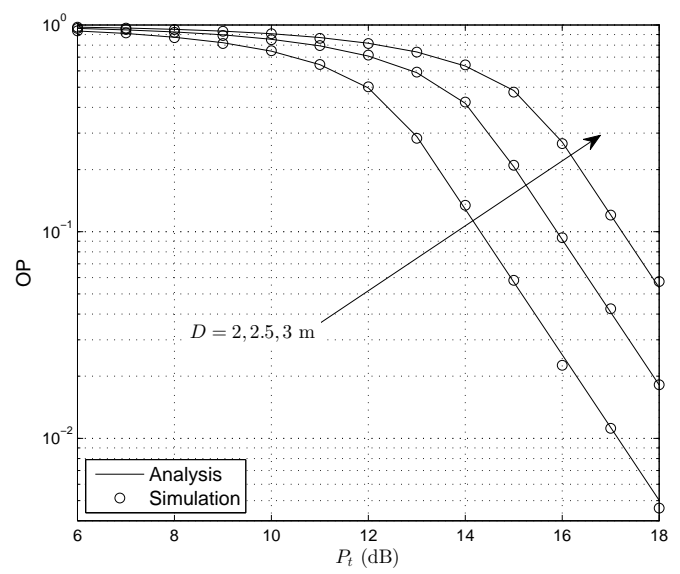

Fig. 4: OP vs. $P_{t}$ for various $D$

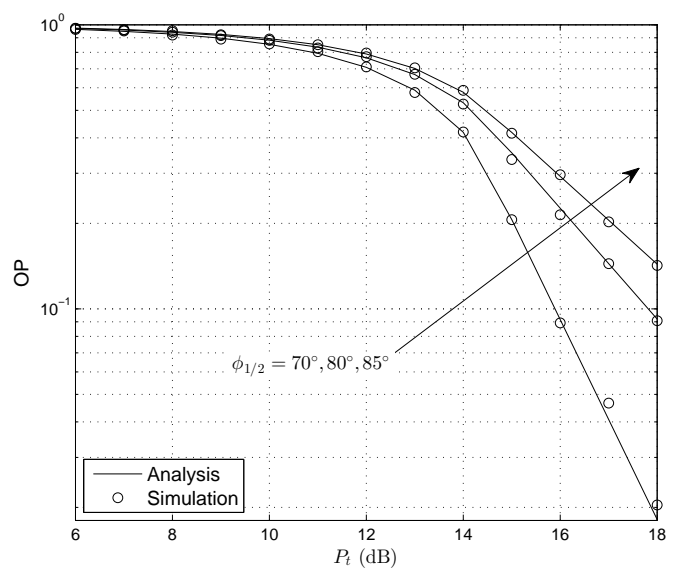

Fig. 5: OP vs. $P_{t}$ for various $\phi_{1 / 2}$

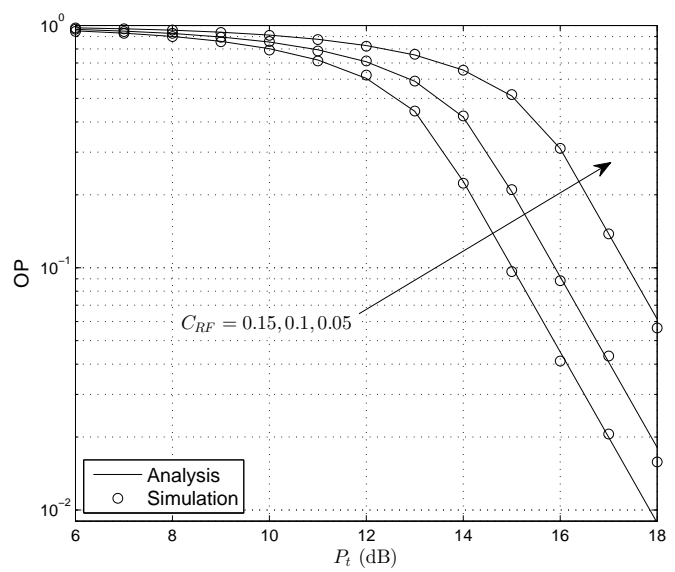

Fig. 6: OP vs. $P_{t}$ for various $C_{R F}$

\section{A. Outage over the VLC downlink}

As observed in Fig. 4, the OP with a small $D$ outperforms the one with a large $D$, as a large $D$ represents a large 3-D space for the devices to place and further means that large transmission distance suffered by VLC signal, which finally leads to the degraded transmit power over the uplink.

As shown in Fig. 5, one can see that the OP with a small the semiangle of the LED, $\phi_{1 / 2}$, outperforms the one with a large the semiangle of the LED, $\phi_{1 / 2}$. Because a large the semiangle of the LED, $\phi_{1 / 2}$, represents that a small the order of Lambertian emission, $m$, as suggested by the relationship $m=-\ln 2 / \ln \left(\cos \left(\phi_{1 / 2}\right)\right)$ presented in Part A of Section II, leading to an decreased channel gain over the VLC downlink and further degrading the SNR of the received VLC signal at the device.

Obviously, as depicted in Fig. 6, the OP with a large $C_{R F}$ outperforms the one with a small $C_{R F}$. It can be explained the fact that: A large $C_{R F}$ represents a large power constant at the devices. Then, under this case more RF power can be converted for the data decoding with an improved transmit SNR.

As presented in Figs. 4-5, we can easily find that the size of the room $(D)$ and the semiangle of the LED $\left(\phi_{1 / 2}\right.$ exhibit a negative effect on the outage performance of the data transmission over the VLC downlink, because they can degrade the received power over the VLC downlink. Moreover, it is easy to observe that simulation and analysis results match very well with each other, which verify the correctness of the proposed analytical model.

\section{B. Outage for the RF uplink}

In this subsection, some parameters are set as: $P_{t}=10$ $\mathrm{W}, L=4, T_{u p}=5 \mathrm{~s}, T_{u p} / T_{d n}=10^{-5}, \gamma_{t h}=-10$ $\mathrm{dB}$ and the truncation factor adopt for (47) and (48) is set as $10^{7}$. As the main focuses of this work is analyzing the outage performance of the target system, in the following user pairing is ignored and only two devices are considered with the purpose of simplifying the realizations of the target system.

As shown by the derived light EH model, (12) in Part B of Section III, the number of LED lamps, $L$, exhibits a similar positive influence on the light $\mathrm{EH}$, with some other systems parameters, e.g., the power constant, $C_{R F}$, and the $\mathrm{EH}$ efficiency, $\rho_{j}$, and the transmit power at LED lamps, $P_{t}$. Moreover, the size of the room, $D$, determines the distribution range of the devices, namely, the value of $d_{j}$ in (12), which show a negative effect on light EH. Therefore, we will indirectly investigate the impacts of light $\mathrm{EH}$ on the outage performance of the information deliveries over the RF uplink via changing values of $L$ and $D$, as shown in Figs. 8 and 9.

As depicted in Fig. 7-9, it is clear that the OP for device 1 gets worse slowly and the one for device 2 gets better quickly, while increasing $P_{t g}$. Because under NOMA scheme

\footnotetext{
${ }^{7}$ In the following three figures, OP lines are depicted with the parameters in a vector, the order of which follows the arrow direction, e.g., in Fig. 7, the top and bottom black lines are respectively with $\gamma_{t h}=0 \mathrm{~dB}$ and $\gamma_{t h}=-10$ $\mathrm{dB}$, while the top and bottom red lines are respectively with $\gamma_{t h}=-10 \mathrm{~dB}$ and $\gamma_{t h}=0 \mathrm{~dB}$.
} 


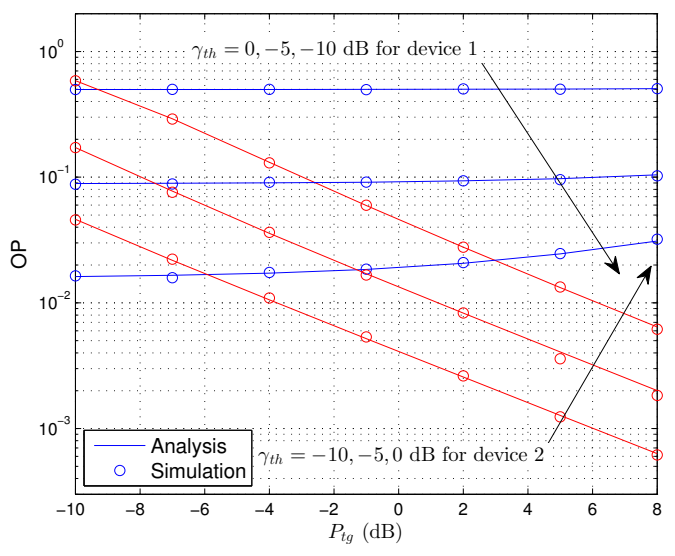

Fig. 7: OP vs. $P_{t g}$ for various $\gamma_{t h}$

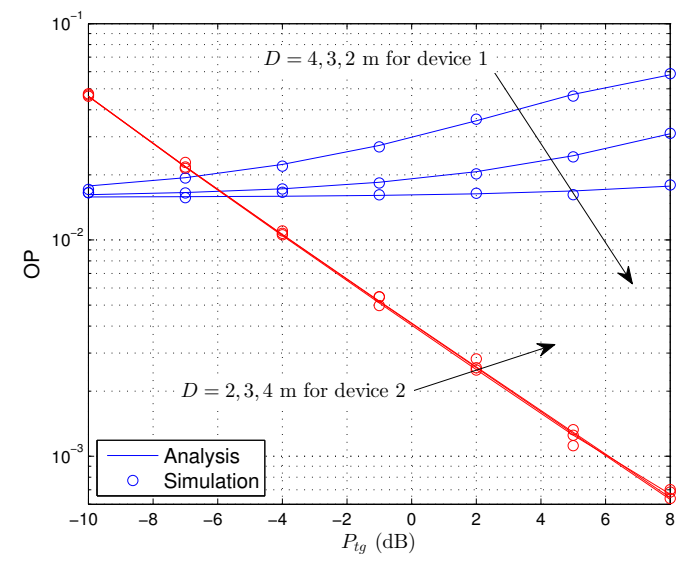

Fig. 8: OP vs. $P_{t g}$ for various $D$

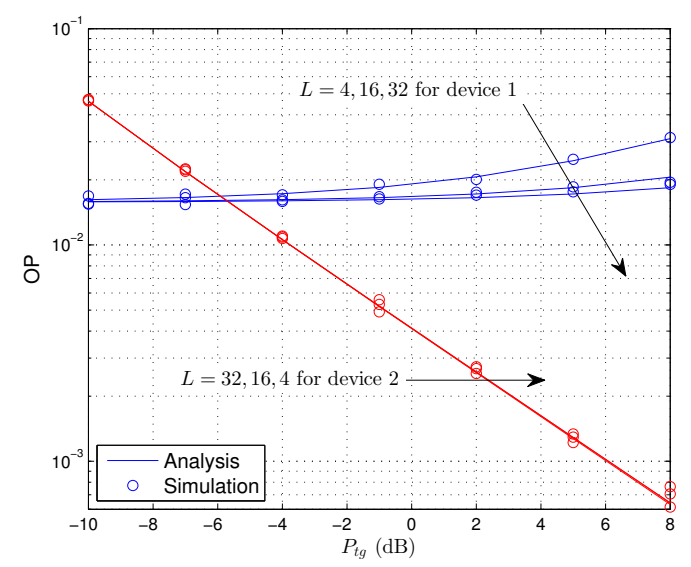

Fig. 9: OP vs. $P_{t g}$ for various $L$

a higher $P_{t g}$ represents that a higher transmit power is needed for device 2 to promise its data transmission over the uplink, resulting in an increased interference to the received signal for device 1 at the RF receiver, as shown by (25). The negative effect of the interference arisen from device 2 on the received SINR of device 1 outperforms the positive effect of increasing $P_{t g}$ on the transmit power at device 1, thus leading to the degraded OP for device 1 .

Therefore, we can draw a conclusion as follows: Under NOMA scheme the improvement on the performance of device 2 comes at the cost of sacrificing part of the performance of device 1. Furthermore, it is clear that the simulation and analytical results match well with each other, which verify our proposed analytical models.

In detail, we can observe from Fig. 7 that a lower $\gamma_{t h}$ leads to a lower OP for both of the two devices, as a lower $\gamma_{t h}$ means a lower probability for the received SINR drops below the threshold. In Figs. 7 and 8, it is clear that, for device 1, the OP with a smaller $D$ or a larger $L$ outperforms the one with a lager $D$ or a smaller $L$, respectively.

These two observations can be respectively explained by the following facts: 1) A larger $D$ means that there is more space for the devices to distribute, then the received signal suffers a larger pathloss; 2) A larger $L$ means that more energy can be harvested for the transmission over the uplink, thus leading to an improved received SINR at the RF receiver.

Furthermore, we can also find from Figs. 8 and 9 that device 2 exhibits a similar OP for various of $D / L$. It is because of the fact: when NOMA scheme is adopted for the uplink transmission, the targeted performance of device 2, which suffers a deeper fading (including small-scale fading and pathloss), is firstly promised while setting the transmit power as suggested by (21)-(23).

\section{CONCLUSION}

In this paper, we have studied the outage performance of a 3-D hybrid VLC-RF indoor IoT system, while considering the randomness of the positions of the devices and light EH. VLC is considered for the downlink from the lamps to the devices, while traditional RF transmission technology with NOMA scheme is employed over the uplink. The approximated analytical expressions for the outage probability over the VLC downlink and the RF uplink are respectively derived and verified via Monte-Carlo simulations. Our proposed analytical models can be useful for analyzing effects of system parameters, e.g., the transmit power, room size, the distribution density of the devices and the number of the antennas, on outage performance and can be effective for solving various optimization and approximation tasks of the considered system.

\section{APPENDIX}

\section{A. Proof of Theorem 1}

By using (1), we can obtain the CDF of $d_{i}$ and $\phi_{i}(i=1,2)$ as

$$
\begin{aligned}
F_{d_{i}}(x) & =\int_{0}^{x} \int_{0}^{\pi / 2} \int_{0}^{2 \pi} \frac{3}{2 \pi D^{3}} \sin \phi_{i} d_{i}^{2} d \theta_{i} d \phi_{i} d\left(d_{i}\right) \\
& = \begin{cases}0, & x<0 \\
\frac{x^{3}}{D^{3}}, & 0 \leq x \leq D \\
1, & x>D\end{cases}
\end{aligned}
$$


and

$$
\begin{aligned}
F_{\phi_{i}}(x) & =\int_{0}^{D} \int_{0}^{x} \int_{0}^{2 \pi} \frac{3}{2 \pi D^{3}} \sin \phi_{i} d_{i}^{2} d \theta_{i} d \phi_{i} d\left(d_{i}\right) \\
& = \begin{cases}0, & x<0 \\
1-\cos x, & 0 \leq x \leq \frac{\pi}{2} \\
1, & x>\frac{\pi}{2}\end{cases}
\end{aligned}
$$

respectively.

Then, it is to easy obtain the PDF of $d_{i}$ and $\phi_{i}(i=$ $1,2)$ as $f_{d_{i}}(x)=\left\{\begin{array}{ll}\frac{3 x^{2}}{D^{3}}, & \text { if } 0 \leq x \leq D \\ 0, & \text { else }\end{array}\right.$ and $f(x)=$ $\left\{\begin{array}{ll}\sin x, & \text { if } 0 \leq x \leq \frac{\pi}{2} \\ 0, & \text { else }\end{array}\right.$, respectively.

Let $Z=\cos ^{p}\left(\phi_{i}\right)$, where $p$ is a non-zero constant, then $\phi_{i}=\arccos \left(Z^{1 / p}\right)$. Therefore, the PDF of $Z$ can be given as

$$
\begin{aligned}
f_{Z}(z) & =\sin \left(\arccos \left(z^{1 / p}\right)\right) \times\left|-\frac{1}{\sqrt{1-z^{2 / p}}} \cdot \frac{1}{p} z^{\frac{1}{p}-1}\right| \\
& =\frac{1}{p} z^{\frac{1}{p}-1} .
\end{aligned}
$$

Thus, we can also easily obtain the $\mathrm{CDF}$ of $Z$ as $F_{Z}(x)=$ $z^{\frac{1}{p}}$.

In the following, we will present the derivation of Theorem 1 .

Using (49), we can have

$$
\begin{aligned}
I & =\operatorname{Pr}\left\{d_{i}^{p} \leq a \cos ^{q}\left(\phi_{i}\right)\right\} \\
& =1-\int_{0}^{D} F_{X}(x) \frac{3 x^{2}}{D^{3}} d x,
\end{aligned}
$$

where $X=a^{\frac{1}{p}} \cos ^{\frac{q}{p}}\left(\phi_{i}\right)$.

Making of the CDF of $Z=\cos ^{p}\left(\phi_{i}\right)$, we can have

$$
\begin{aligned}
F_{X}(x) & =\operatorname{Pr}\left\{\cos \left(\phi_{2}\right)<\left(\frac{x^{p}}{a}\right)^{\frac{1}{q}}\right\} \\
& = \begin{cases}1, & x>a^{\frac{1}{p}} \\
a^{-\frac{1}{q}} x^{\frac{p}{q}}, & \text { else }\end{cases}
\end{aligned}
$$

Then, substituting (53) into (52), we can finally achieve

$$
\begin{aligned}
I & =1-\int_{0}^{D} F_{X}(x) \frac{3 x^{2}}{D^{3}} d x \\
& = \begin{cases}1-\frac{3 q}{3 q+p} a^{-\frac{1}{q}} D^{\frac{p}{q}}, & \text { if } a^{\frac{1}{p}} \geq D \\
\frac{p}{3 q+p} \frac{1}{D^{3}} a^{\frac{3}{p}}, & \text { else }\end{cases}
\end{aligned}
$$

Then, the proof is completed.

\section{B. Proof of Theorem 2}

Using (49), we can write $T=\operatorname{Pr}\left\{d_{2} \leq a d_{1} \frac{\cos ^{p}\left(\phi_{2}\right)}{\cos ^{q}\left(\phi_{1}\right)}\right\}$ ( $a$, $p$ and $q$ are positive constants) as

$$
\begin{aligned}
T & =1-\operatorname{Pr}\left\{X \leq d_{2}\right\} \\
& =1-\frac{3}{D^{3}} \int_{0}^{D} F_{X}\left(d_{2}\right) x^{2} d x,
\end{aligned}
$$

where $X=d_{1} a \frac{\cos ^{p}\left(\phi_{2}\right)}{\cos ^{q}\left(\phi_{1}\right)}$ and $F_{X}(x)$ is the CDF of $X$.

Using the definition of CDF and (50), we can obtain

$$
\begin{aligned}
F_{X}(x) & =\operatorname{Pr}\{\cos \left(\phi_{2}\right)<\underbrace{\left(\frac{x}{d_{1}}\right)^{\frac{1}{p}} a^{-\frac{1}{p}} \cos ^{\frac{q}{p}}\left(\phi_{1}\right)}_{Y}\} \\
& =1-\int_{0}^{1} F_{Y}(y) d y,
\end{aligned}
$$

where $F_{Y}(y)$ is the CDF of $Y$.

Similar to the derivation of (56), we can also have

$$
\begin{aligned}
F_{Y}(y) & =\operatorname{Pr}\{\cos \left(\phi_{1}\right) \leq \underbrace{y^{\frac{p}{q}}\left(\frac{d_{1}}{x}\right)^{\frac{1}{q}} a^{\frac{1}{q}}}_{Z}\} \\
& =1-\int_{0}^{1} F_{Z}(z) d z,
\end{aligned}
$$

where $F_{Z}(z)$ is the $\mathrm{CDF}$ of $Z$.

Making use of the PDF of $d_{1}$, we can obtain

$$
\begin{aligned}
F_{Z}(z) & =\operatorname{Pr}\left\{y^{\frac{p}{q}}\left(\frac{d_{1}}{x}\right)^{\frac{1}{q}} a^{\frac{1}{q}} \leq z\right\} \\
& =\left\{\begin{array}{ll}
1, & \text { if } z \geq\left(\frac{\tau}{x}\right)^{\frac{1}{q}} y^{\frac{p}{q}} \\
\left(\frac{x}{\tau}\right)^{3} \frac{z^{3 q}}{y^{3 p}}, & \text { else }
\end{array},\right.
\end{aligned}
$$

where $\tau=a D$.

Using (58) in (57), we can obtain

$$
\begin{aligned}
F_{Y}(y) & =1-\int_{0}^{1} F_{Z}(z) d z \\
& = \begin{cases}1-\frac{1}{1+3 q}\left(\frac{x}{\tau}\right)^{3} y^{-3 p}, & \text { if } y>\Pi_{2} \\
\frac{3 q}{1+3 q}\left(\frac{\tau}{x}\right)^{\frac{1}{q}} y^{\frac{p}{q}}, & \text { else }\end{cases}
\end{aligned}
$$

where $\Pi_{1}=\left(\frac{\tau}{x}\right)^{\frac{1}{q}} y^{\frac{p}{q}}$ and $\Pi_{2}=\left(\frac{x}{\tau}\right)^{\frac{1}{p}}$.

Using (59) in (56), we can obtain

$$
\begin{aligned}
F_{X}(x) & =1-\int_{0}^{1} F_{Y}(y) d y \\
& = \begin{cases}1-\mathrm{A}\left(\frac{\tau}{x}\right)^{\frac{1}{q}}, & \text { if } x>\tau \\
\mathrm{B}\left(\frac{x}{\tau}\right)^{\frac{1}{p}}-\mathrm{C}\left(\frac{x}{\tau}\right)^{3}, & \text { else }\end{cases}
\end{aligned}
$$

where $\mathrm{A}=\frac{3 q}{1+3 q} \frac{q}{p+q}, \mathrm{~B}=1-\mathrm{A}-\mathrm{C}$ and $\mathrm{C}=\frac{1}{1+3 q} \frac{1}{1-3 p}$.

Finally, using (60) in (55), we can obtain $I_{P_{22}}$ as

$$
\begin{aligned}
T & =1-\int_{0}^{D} F_{X}(x) \frac{3 x^{2}}{D^{3}} d x \\
& =\left\{\begin{array}{ll}
1-\frac{3 p \mathrm{~B}}{1+3 p} a^{-\frac{1}{p}}-\frac{\mathrm{C}}{2} a^{-3}, \\
\left(1-\frac{\mathrm{C}}{2}-\frac{3 p \mathrm{~B}}{1+3 p}-\frac{3 q \mathrm{~A}}{3 q-1}\right) a^{3}+\frac{3 q \mathrm{~A}}{3 q-1} a^{\frac{1}{q}}, & \text { else }
\end{array} .\right.
\end{aligned}
$$

Then, the proof is completed. 


\section{Proof of Theorem 3}

According to [43], Marcum $Q$-function can be presented in terms of generalized Laguerre polynomials as

$Q_{1}(a, b)=1-\sum_{k=0}^{\infty}(-1)^{k} \exp \left(-\frac{a^{2}}{2}\right) \frac{L_{k}^{(0)}\left(\frac{a^{2}}{2}\right)}{\Gamma(k+2)}\left(\frac{b^{2}}{2}\right)^{k+1}$,

where $L_{k}^{(0)}(x)=\sum_{l=0}^{k} \frac{k !}{(k-l) !(l !)^{2}}(-x)^{l}$ is the generalized Laguerre polynomial with degree $k$ and order 0 , given by Eq. (22.3.9) in [44].

Using (62), we can calculate $I=\operatorname{Pr}\left\{\frac{a}{d^{q}} \cos ^{p}(\phi)|h|^{2} \leq b\right\}$ as

$$
\begin{aligned}
I= & \operatorname{Pr}\left\{|h|^{2} \leq \frac{b}{a} \frac{d^{q}}{\cos ^{p}(\phi)}\right\} \\
= & 1-Q_{1}\left(\sqrt{2 K}, \sqrt{\frac{2(1+K)\left(\frac{b}{a} \frac{d^{q}}{\cos ^{p}(\phi)}\right)}{\Omega}}\right) \\
= & \sum_{k=0}^{\infty}(-1)^{k} \exp (-K) \frac{L_{k}^{(0)}(K)}{\Gamma(k+2)} \\
& \cdot\left(\frac{b(1+K)}{a \Omega}\right)^{k+1} \frac{d^{q(k+1)}}{\cos ^{p(k+1)}(\phi)} .
\end{aligned}
$$

Considering the randomness of the distribution of the device and (18), we can further calculate (63) as

$$
\begin{aligned}
I= & \beta \sum_{k=0}^{\infty}(-1)^{k} \exp (-K) \frac{L_{k}^{(0)}(K)}{\Gamma(k+2)}\left(\frac{b(1+K)}{a \Omega}\right)^{k+1} \\
& \cdot \int_{0}^{D} \int_{0}^{\pi / 2} \int_{0}^{2 \pi} \frac{\sin (\phi)}{\cos ^{p(k+1)}(\phi)} d^{q(k+1)+2} d(\theta) d(\phi) d(d) \\
= & 3 \sum_{k=0}^{\infty}(-1)^{k} \exp (-K) \frac{L_{k}^{(0)}(K)}{\Gamma(k+2)} \\
& \cdot\left(\frac{b(1+K)}{a \Omega}\right)^{k+1} \frac{D^{q(k+1)}}{q(k+1)+3} \cdot \frac{1}{1-p(k+1)} .
\end{aligned}
$$

Then, the proof is completed.

\section{REFERENCES}

[1] S. Rajagopal, R. D. Roberts, and S.-K. Lim, "IEEE 802.15.7 visible light communication: Modulation schemes and dimming support," IEEE Commun. Mag., vol. 50, no. 3, pp. 72-82, Mar. 2012.

[2] H. Elgala, R. Mesleh, and H. Haas, "Indoor optical wireless communication: Potential and state-of-the-art," IEEE Commun. Mag., vol. 49, no. 9, pp. 56-62, Sept. 2011.

[3] J. Ye, H. Lei, Y. Liu, G. Pan, D. B. da Costa, Q. Ni, Z. Ding, "Cooperative communications with wireless energy harvesting over nakagami- $m$ fading channels," IEEE Trans. Commun., vol. 65, no. 12, pp. 5149-5164, Dec. 2017.

[4] A. Siddiqui, L. Musavian, S. Aissa, Q. Ni "Performance analysis of relaying systems with fixed and energy harvesting batteries," IEEE Trans. Commun., vol. 66, no. 4, pp. 1386-1398, April 2018.

[5] F. K. Shaikh, S. Zeadally, "Energy harvesting in wireless sensor networks: A comprehensive review," Renew. Sust. Energ. Rev., vol. 55, pp. 10411054, Mar. 2016.

[6] A. Nasiri, S. A. Zabalawi and G. Mandic, "Indoor power harvesting using photovoltaic cells for low-power applications," IEEE Trans. Ind. Electron., vol. 56, no. 11, pp. 4502-4509, Nov. 2009.
[7] G. Pan, P. D. Diamantoulakis, Z. Ma, Z. Ding and G. K. Karagiannidis, "Simultaneous lightwave information and power transfer: Policies, techniques and future directions," IEEE Access, vol. 7, pp. 28250-28257, Feb. 2019.

[8] C. Carvalho, N. Paulino, "On the feasibility of indoor light energy harvesting for wireless sensor networks," Procedia Technology, vol. 17, pp. 343-350, 2014

[9] H. Yu, Q. Yue, "Indoor light energy harvesting system for energy-aware wireless sensor node," Energy Procedia, vol. 16, Part B, pp. 1027-1032, 2012.

[10] Z. Wang, D. Tsonev, S. Videv and H. Haas, "On the design of a solarpanel receiver for optical wireless communications with simultaneous energy harvesting," IEEE J. Sel. Areas in Commun., vol. 33, no. 8, pp. 1612-1623, Aug. 2015

[11] C. Li, W. Jia, Q. Tao, and M. Sun, "Solar cell phone charger performance in indoor environment," in Proc. IEEE 37th Annu. Northeast Bioeng. Conf. (NEBEC), Troy, NY, USA, Apr. 2011, pp. 1-2.

[12] A. Dionisi, D. Marioli, E. Sardini and M. Serpelloni, "Autonomous wearable system for vital signs measurement with energy-harvesting module," IEEE Trans. Instrum. Meas., vol. 65, no. 6, pp. 1423-1434, June 2016.

[13] J. W. Kimball, B. T. Kuhn and R. S. Balog, "A system design approach for unattended solar energy harvesting supply," IEEE Trans. Power Electron., vol. 24, no. 4, pp. 952-962, Apr. 2009.

[14] E. G. Fong, N. J. Guilar, T. J. Kleeburg, H. Pham, D. R. Yankelevich and R. Amirtharajah, "Integrated energy-harvesting photodiodes with diffractive storage capacitance," IEEE Trans. Very Large Scale Integr. (VLSI) Syst., vol. 21, no. 3, pp. 486-497, Mar. 2013.

[15] G. Moayeri Pour, M. K. Benyhesan and W. D. Leon-Salas, "Energy harvesting using substrate photodiodes," IEEE Trans. Circuits Syst. II, Exp. Briefs, vol. 61, no. 7, pp. 501-505, July 2014.

[16] A. S. Teran, E. Moon, W. Lim, G. Kim, I. Lee, D. Blaauw, J. D. Phillips, "Energy harvesting for GaAs photovoltaics under low-flux indoor lighting conditions," IEEE Trans. Electron. Devices, vol. 63, no. 7, pp. 2820-2825, July 2016.

[17] P. D. Diamantoulakis, G. K. Karagiannidis and Z. Ding, "Simultaneous lightwave information and power transfer (SLIPT)," IEEE Trans. Green Commun. and Net., vol. 2, no. 3, pp. 764-773, Sept. 2018.

[18] T. Rakia, H. C. Yang, F. Gebali and M. S. Alouini, "Optimal design of dual-hop VLC/RF communication system with energy harvesting," IEEE Commun. Lett., vol. 20, no. 10, pp. 1979-1982, Oct. 2016.

[19] G. Pan, J. Ye, Z. Ding, "Secure hybrid VLC-RF systems with light energy harvesting", IEEE Trans. Commun., vol. 65, no. 10, pp. 43484359, Oct. 2017.

[20] Q. Huang, C. Lu, and M. Shaurette, "Feasibility study of indoor light energy harvesting for intelligent building environment management," in Proc. International High Performance Buildings Conference 2010, West Lafayette, Indiana, USA, Paper 18, July 2010.

[21] Q. Huang, X. Li, M. Shaurette, "Integrating Li-Fi wireless communication and energy harvesting wireless sensor for next generation building management," in Proc. International High Performance Building Conference, 2014, West Lafayette, Indiana, USA, Paper 120, July 2014.

[22] M. Ayyash, et al., "Coexistence of WiFi and LiFi toward 5G: Concepts, opportunities, and challenges," IEEE Coтmu. Mag., vol. 54, no. 2, pp. 64-71, Feb. 2016.

[23] D. A. Basnayaka and H. Haas, "Hybrid RF and VLC systems: Improving user data rate performance of VLC systems," in Proc. 2015 IEEE 81st Vehicular Technology Conference (VTC Spring), Glasgow, 2015, pp. 1-5.

[24] S. Shao, et al., "Design and analysis of a visible-light-communication enhanced WiFi system," IEEE/OSA J. Opt. Commun. Netw., vol. 7, no. 10, pp. 960-973, Oct. 2015.

[25] C. Zhang, J. Ye, G. Pan and Z. Ding, "Cooperative hybrid VLC-RF systems with spatially random terminals," IEEE Trans. Commun., vol. 66, no. 12, pp. 6396 - 6408, Dec. 2018

[26] G. Pan, H. Lei, Z. Ding and Q. Ni, "On 3-D hybrid VLC-RF systems with light energy harvesting and OMA scheme over RF links," in Proc. IEEE GLOBECOM 2017, Singapore, 2017, pp. 1-6.

[27] G. Pan, J. Ye, Z. Ding, "On secure VLC systems with spatially random terminals," IEEE Commun. Lett., vol. 21, no. 3, pp. 492-495, Mar. 2017.

[28] H. Wang, S. Ma, T.-S. Ng, and H. V. Poor, "A general analytical approach for opportunistic cooperative systems with spatially random relays," IEEE Trans. Wireless Commun., vol. 10, no. 12, pp. 4122-4129, Dec. 2011.

[29] J. M. Kahn and J. R. Barry, "Wireless infrared communications," Proc. IEEE, vol. 85, no. 2, pp. 265-298, Feb. 1997. 
[30] F. R. Gfeller and U. Bapst, "Wireless in-house data communication via diffuse infrared radiation," Proc. IEEE, vol. 67, no. 11, pp. 1474-1486, Nov. 1979.

[31] P. H. Pathak, X. Feng, P. Hu and P. Mohapatra, "Visible light communication, networking, and sensing: A survey, potential and challenges," IEEE Commun. Surveys Tuts., vol. 17, no. 4, pp. 2047-2077, Fourth quarter 2015.

[32] H. S. Kim, D. R. Kim, S. H. Yang, Y. H. Son and S. K. Han, "An indoor visible light communication positioning system using a RF carrier allocation technique," J. Lightw. Technol., vol. 31, no. 1, pp. 134-144, Jan. 2013.

[33] S. H. Yang, E. M. Jung and S. K. Han, "Indoor location estimation based on LED visible light communication using multiple optical receivers," IEEE Commu. Lett., vol. 17, no. 9, pp. 1834-1837, Sept. 2013.

[34] C. R. Valenta and G. D. Durgin, "Harvesting wireless power: Survey of energy-harvester conversion efficiency in far-field, wireless power transfer systems," IEEE Microw. Mag., vol. 15, no. 4, pp. 108-120, June 2014.

[35] S. Wyne, A. P. Singh, F. Tufvesson and A. F. Molisch, "A statistical model for indoor office wireless sensor channels," IEEE Trans. Wireless Commun., vol. 8, no. 8, pp. 4154-4164, Aug. 2009.

[36] I. Dey, G. G. Messier and S. Magierowski, "Joint fading and shadowing model for large office indoor WLAN environments," IEEE Trans. Antennas Propag., vol. 62, no. 4, pp. 2209-2222, Apr. 2014.

[37] N. Zhang, J. Wang, G. Kang and Y. Liu, "Uplink nonorthogonal multiple access in 5G systems," IEEE Commun. Lett., vol. 20, no. 3, pp. 458-461, Mar. 2016.

[38] Z. Ding, P. Fan and H. V. Poor, "Impact of user pairing on 5G nonorthogonal multiple-access downlink transmissions," IEEE Trans. Veh. Tech., vol. 65, no. 8, pp. 6010-6023, Aug. 2016.

[39] Z. Ding, M. Peng and H. V. Poor, "Cooperative non-orthogonal multiple access in 5G systems," IEEE Commun. Lett., vol. 19, no. 8, pp. 14621465, Aug. 2015.

[40] L. Zeng et al., "High data rate multiple input multiple output (MIMO) optical wireless communications using white LED lighting, IEEE J. Sel. Areas Сотти., vol. 27, no. 9, pp. 1654-1662, 2009.

[41] G. Shi, K. Li, Signal Interference in WiFi and ZigBee Networks, Springer International Publishing, 2017, pp. 34.

[42] Nathan Pletcher and Jan M. Rabaey, "Ultra-low power wake-up receivers for wireless sensor networks," Ph.D. dissertation, EECS Department, University of California, Berkeley, CA, USA, 2008.

[43] S. Andras, A. Baricz, and Y. Sun, "The generalized Marcum $Q$-function: An orthogonal polynomial approach," Acta Uni. Sapientiae Mathem., vol. 3, pp. 60-76, 2011.

[44] M. Abramowitz and I. A. Stegun, Eds., Handbook of Mathematical Functions with Formulas, Graphs, and Mathematical Tables, 10th ed. Dover Publications, 1972.

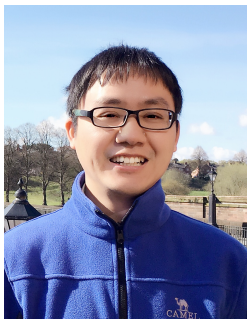

Gaofeng Pan (M'12) received his B.Sc in Communication Engineering from Zhengzhou University, Zhengzhou, China, in 2005, and the Ph.D. degree in Communication and Information Systems from Southwest Jiaotong University, Chengdu, China, in 2011.

He was with The Ohio State University, Columbus, OH, USA, from Sept. 2009 to April 2011 as a joint-trained $\mathrm{PhD}$ student under the supervision of Prof. Eylem Ekici. From May 2012 to April 2019, he was with Southwest University, Chongqing, China, and he was also with School of Computing and Communications, Lancaster University, Lancaster, U.K., from Jan. 2016 to Jan. 2018, where he was a postdoc under the supervision of Prof. Zhiguo Ding. Since April 2019, he has been with School of Information and Electronics, Beijing Institute of Technology, P. R. China, as a professor. His research interest spans special topics in communications theory, signal processing and protocol design, in cluding visible light communications, secure communications, $\mathrm{CR} /$ cooperative communications, UAV communications and MAC protocols. He has served as a reviewer for major international journals, e.g., IEEE Journal of Selected Areas in Communications, IEEE Communications Magazine, IEEE Transactions on Communications, IEEE Transactions on Wireless Communications, IEEE Transactions on Signal Processing, IEEE Transactions on Vehicular Technology, etc. He received the Exemplary Reviewer Award 2017 given by IEEE Transactions on Communications.

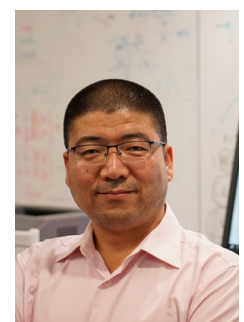

Hongjiang Lei (M'17) received the B.Sc. degree in Mechanical and Electrical Engineering from Shenyang Institute of Aeronautical Engineering, Shenyang, China, in 1998, the M.Sc. degree in Computer Application Technology from Southwest Jiaotong University, Chengdu, China, in 2004, and the Ph.D. degree in Instrument Science and Technology from Chongqing University, Chongqing, China, in 2015, respectively. In May 2004, he joined the School of Communication and Information Engineering (SCIE) of Chongqing University of Posts and Telecommunications (CQUPT), Chongqing, China, where he is currently an Associate Professor. From November 2016 to October 2018, he was a Postdoctoral Research Fellow with CEMSE Division, King Abdullah University of Science and Technology (KAUST), Saudi Arabia. His current research interests include physical layer security, cooperative relaying systems, and cognitive radio networks. He is a TPC Member of IEEE Globecom'2017, 2018, 2019, etc. He has also served as a reviewer for major international journals, e.g., IEEE JSAC, IEEE JSTSP, IEEE TVT, IEEE TCOM, IEEE TWC, IEEE TIFS, IEEE WCL, IEEE CL, etc.

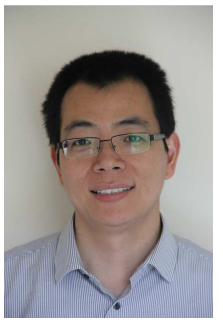

Zhiguo Ding (S'03-M'05-SM'15) received his B.Eng in Electrical Engineering from the Beijing University of Posts and Telecommunications in 2000, and the Ph.D degree in Electrical Engineering from Imperial College London in 2005. From Jul. 2005 to Apr. 2018, he was working in Queen's University Belfast, Imperial College, Newcastle University and Lancaster University. Since Apr. 2018, he has been with the University of Manchester as a Professor in Communications. From Oct. 2012 to Sept. 2018 he has also been an academic visitor in Princeton

University.

Dr Ding's research interests are 5G networks, game theory, cooperative and energy harvesting networks and statistical signal processing. He is serving as an Editor for IEEE Transactions on Communications, IEEE Transactions on Vehicular Technology, and Journal of Wireless Communications and Mobile Computing, and was an Editor for IEEE Wireless Communication Letters, IEEE Communication Letters from 2013 to 2016. He received the best paper award in IET ICWMC-2009 and IEEE WCSP-2014, the EU Marie Curie Fellowship 2012-2014, the Top IEEE TVT Editor 2017, IEEE Heinrich Hertz Award 2018 and the IEEE Jack Neubauer Memorial Award 2018.

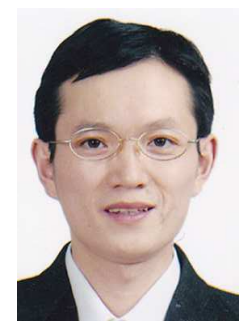

Qiang Ni (M'04-SM'08) received the B.Sc., M.Sc., and Ph.D. degrees from the Huazhong University of Science and Technology, China, all in engineering. $\mathrm{He}$ is currently a Professor and the Head of the Communication Systems Group, School of Computing and Communications, Lancaster University, Lancaster, U.K. His research interests include the area of future generation communications and networking, including green communications and networking, millimeter-wave wireless communication$\mathrm{s}$, cognitive radio network systems, non-orthogonal multiple access (NOMA), heterogeneous networks, 5G and 6G, SDN, cloud networks, energy harvesting, wireless information and power transfer, IoTs, cyber physical systems, machine learning, big data analytics, and vehicular networks. He has authored or co-authored over 200 papers in these areas. He was an IEEE 802.11 Wireless Standard Working Group Voting Member and a contributor to the IEEE Wireless Standards. 